Geometry $\&$ Topology

Volume 8 (2004) 701-734

Published: 16 May 2004

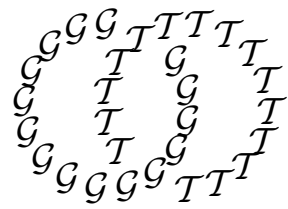

\title{
Orbifold adjunction formula and symplectic cobordisms between lens spaces
}

\author{
Weimin Chen \\ Mathematics Department, Tulane University \\ New Orleans, LA 70118, USA \\ Email: wchen@math.tulane.edu
}

\begin{abstract}
Each lens space has a canonical contact structure which lifts to the distribution of complex lines on the three-sphere. In this paper, we show that a symplectic homology cobordism between two lens spaces, which is given with the canonical contact structure on the boundary, must be diffeomorphic to the product of a lens space with the unit interval. As one of the main ingredients in the proof, we also derive in this paper the adjunction and intersection formulae for pseudoholomorphic curves in an almost complex 4-orbifold, extending the relevant work of Gromov and McDuff in the manifold setting.
\end{abstract}

\section{AMS Classification numbers Primary: 57R17}

Secondary: $57 \mathrm{R} 80$

Keywords: Cobordism of lens spaces, orbifold adjunction formula, symplectic 4-orbifolds, pseudoholomorphic curves

Proposed: Yasha Eliashberg

Seconded: Robion Kirby, Ronald Fintushel
Received: 27 December 2003

Revised: 20 January 2004 


\section{Introduction}

In this paper, we prove the following theorem.

Theorem 1.1 Let $(W, \omega)$ be a symplectic homology cobordism between two lens spaces which are equipped with their canonical contact structure. Then $W$ is diffeomorphic to the product of a lens space with the unit interval.

Here the canonical contact structure $\xi_{0}$ on a lens space $L(p, q)$ is the descendant of the distribution of complex lines on $\mathbb{S}^{3}=\left\{\left.\left(z_{1}, z_{2}\right)|| z_{1}\right|^{2}+\left|z_{2}\right|^{2}=1\right\}$ under the quotient map $\mathbb{S}^{3} \rightarrow L(p, q)$ of the $\mathbb{Z}_{p}$-action $\left(z_{1}, z_{2}\right) \mapsto\left(\mu_{p} z_{1}, \mu_{p}^{q} z_{2}\right)$. (Here $\mu_{p}=\exp \left(\sqrt{-1} \frac{2 \pi}{p}\right)$, and $p, q$ are relatively prime and $0<q<p$.) The contact structure $\xi_{0}$ induces a canonical orientation on $L(p, q)$ where a volume form is given by $\alpha \wedge d \alpha$ for some 1 -form $\alpha$ such that $\xi_{0}=\operatorname{ker} \alpha$. A symplectic cobordism from $\left(L\left(p^{\prime}, q^{\prime}\right), \xi_{0}^{\prime}\right)$ to $\left(L(p, q), \xi_{0}\right)$ is a symplectic 4 -manifold $(W, \omega)$ with boundary $\partial W=L(p, q)-L\left(p^{\prime}, q^{\prime}\right)$, such that there exists a vectorfield $v$ in a neighborhood of $L(p, q) \cup L\left(p^{\prime}, q^{\prime}\right) \subset W$, which is transverse to $L(p, q) \cup L\left(p^{\prime}, q^{\prime}\right)$ and for which $L_{v} \omega=\omega, \xi_{0}^{\prime}=\operatorname{ker}\left(\left.i_{v} \omega\right|_{L\left(p^{\prime}, q^{\prime}\right)}\right), \xi_{0}=\operatorname{ker}\left(\left.i_{v} \omega\right|_{L(p, q)}\right)$, and the canonical orientations on $L(p, q), L\left(p^{\prime}, q^{\prime}\right)$ agree with the orientations defined by the normal vector $v$. (Here $W$ is canonically oriented by the symplectic form $\omega$, ie, $\omega \wedge \omega$ is a volume form.) The cobordism $W$ is called a homology cobordism if each $L(p, q) \subset W, L\left(p^{\prime}, q^{\prime}\right) \subset W$ induces an isomorphism on homology groups (with $\mathbb{Z}$ coefficients). In particular, this condition implies $p=p^{\prime}$.

As a special case, consider the following:

Corollary 1.2 Let $\rho$ be a symplectic $\mathbb{Z}_{p}$-action on $\left(\mathbb{R}^{4}, \omega_{0}\right)$ where $\omega_{0}=$ $d x_{1} \wedge d y_{1}+d x_{2} \wedge d y_{2}$. Suppose outside of a ball, $\rho$ is linear and free, and is orthogonal with respect to the Euclidean metric $g_{0}=\sum_{i=1}^{2}\left(d x_{i}^{2}+d y_{i}^{2}\right)$. Then $\rho$ is conjugate to a linear action by a diffeomorphism which is identity outside of a ball.

Remark 1.3 (1) It is likely that Corollary 1.2 can be strengthened to the assertion that the action $\rho$ is conjugate to a linear action by a symplectomorphism of $\left(\mathbb{R}^{4}, \omega_{0}\right)$. We plan to address this problem in a separate paper.

(2) Relevant to Theorem 1.1 and Corollary 1.2, we mention two earlier results. One is due to Eliashberg (cf [6]) which says that a symplectic 4-manifold $W$ with contact boundary $\mathbb{S}^{3}$ (in the weak sense) is diffeomorphic to a blowup of the 4 -ball $\mathbb{B}^{4}$. The other is due to Gromov-McDuff (cf for example Theorem 9.4.2 in [16]) which says that if $(W, \omega)$ is a minimal symplectic $4-$ manifold and 
there are compact subsets $K \subset W$ and $V \subset \mathbb{R}^{4}$ with $V$ being star-shaped such that $(W \backslash K, \omega)$ is symplectormorphic to $\left(\mathbb{R}^{4} \backslash V, \omega_{0}\right)$ via a map $\psi$, then there exists a symplectomorphism $\phi:(W, \omega) \rightarrow\left(\mathbb{R}^{4}, \omega_{0}\right)$ which agrees with $\psi$ on $W \backslash K^{\prime}$ for some larger compact subset $K^{\prime} \supset K$.

(3) Symplectic fillings (in the weak sense) of lens spaces with the canonical contact structure are classified up to orientation-preserving diffeomorphisms in 13, where it is shown that there are infinitely many lens spaces which have a unique filling up to blowups. For these lens spaces, it is clear that when the two ends of $\partial W$ are diffeomorphic, the condition that $W$ is a homology cobordism is equivalent to the condition that $(W, \omega)$ is minimal.

The proof of Theorem 1.1 is based on studying pseudoholomorphic curves in a certain symplectic 4-orbifold in the fashion of Gromov-McDuff in the manifold setting (cf for example [16]). There are two main ingredients. One is the orbifold analog of the adjunction and intersection formulae for pseudoholomorphic curves, extending the relevant work of Gromov and McDuff [7, 14, 15] in the manifold setting. The other is a structural theorem for the space of a certain notion of map 1 between orbifolds developed in [3], which is needed here for the corresponding Fredholm theory.

The paper is organized as follows. In Section 2 we introduce a notion of differentiable chains in orbifolds, which serves as a bridge between the de Rham cohomology of an orbifold and the singular cohomology of its underlying space via integration. Section 3 is devoted to the proof of the orbifold analog of the adjunction and intersection formulae. The main results are proved in Section 4.

\section{Acknowledgments}

I am indebted to Ron Fintushel for bringing the problem of $\mathbb{S}^{1}$-actions on $\mathbb{S}^{5}$ to my attention, and to Sławomir Kwasik for pointing out [17 to me, which eventually led to the study in this paper. I am also very grateful to Dusa McDuff for helpful communications regarding her relevant work and for kindly letting me use [16] before its publication, and to Morris Kalka and Sławomir Kwasik for very valuable conversations. An earlier version of this work contains a serious mistake, thanks to Slava Matveyev for pointing it out to me. Finally, I wish to thank an anonymous referee for pointing out several misspellings in the text and whose queries helped improve the presentation of this article. This research was partially supported by NSF Grant DMS-0304956.

\footnotetext{
${ }^{1} \mathrm{~A}$ prototype of this notion appeared first in 4 in the disguise of "a good (Vmanifold) map + an isomorphism class of the pull-backs of the tangent bundle".
} 


\section{Differentiable chains in orbifolds}

We introduce here a notion of differentiable chains in orbifolds. The homology groups of the corresponding chain complex are naturally isomorphic to the singular homology groups of the underlying space over $\mathbb{Q}$, so that this construction yields an explicit pairing between the de Rham cohomology groups of the orbifold and the singular homology groups of the underlying space via integration over differentiable chains. In light of the development in [3], the notion introduced here may be regarded as a natural generalization to the orbifold category of the notion of differentiable singular chains in smooth manifolds.

A differentiable $r$-chain in an orbifold $X$ (of class $C^{l}$ for some $l \geq 1$ ) is a finite linear combination of differentiable $r$-simplexes in $X$, where a differentiable $r$-simplex $\sigma$ in $X$ is a differentiable map (in the sense of [3]) from a certain $r$-dimensional orbihedron into $X$. More precisely, the said $r$-dimensional orbihedron is an orbispace where the underlying space is the standard $r$-simplex $\Delta^{r}$ in $\mathbb{R}^{r}$, and the orbispace structure is given by a complex of finite groups over $\Delta^{r}$ in the sense of Haefliger [8] (see also Part II of [3]). Recall that a complex of groups consists of the following data: $\left(K, G_{\tau}, \psi_{a}, g_{a, b}\right)$, where $K$ is a simplicial complex, $G_{\tau}$ is a group assigned to each cell $\tau \in K, \psi_{a}: G_{i(a)} \rightarrow G_{t(a)}$ is an injective homomorphism assigned to each edge $a$ in the barycentric subdivision of $K$ with $i(a), t(a)$ being the cells of $K$ whose barycenters are the end points of $a$ such that $t(a)$ is a face of $i(a)$, and $g_{a, b}$ is an element of $G_{t(a)}$ assigned to each pair of composable edges $a, b$ such that

$$
A d\left(g_{a, b}\right) \circ \psi_{a b}=\psi_{a} \circ \psi_{b}, \quad \psi_{a}\left(g_{b, c}\right) g_{a, b c}=g_{a, b} g_{a b, c} .
$$

The orbihedron is covered by a set of "uniformizing systems" which are given with compatible equivariant simplicial structures. The $r$-simplex $\sigma$ being a differentiable map means that the representatives of $\sigma$ are differentiable when restricted to each simplex in the corresponding uniformizing system.

Let $\alpha$ be a differential $r$-form on $X$. Then a differentiable $r$-simplex $\sigma$ in $X$ pulls back $\alpha$ to a differential $r$-form $\sigma^{*} \alpha$ on $\Delta^{r}$, the standard $r$-simplex in $\mathbb{R}^{r}$. We define the integration of $\alpha$ over $\sigma$ by

$$
\int_{\sigma} \alpha=\frac{1}{|G|} \int_{\Delta^{r}} \sigma^{*} \alpha
$$

where $|G|$ is the order of the group $G$ assigned to the top cell of $\Delta^{r}$ in the complex of finite groups that defines the orbispace structure of the orbihedron over which $\sigma$ is defined. The integration over a differentiable $r$-chain $c=$ 
$\sum_{k} a_{k} \sigma_{k}$ is defined to be

$$
\int_{c} \alpha=\sum_{k} a_{k} \int_{\sigma_{k}} \alpha
$$

Next we introduce a boundary operator $\partial$ on the set of differentiable chains. To this end, let $\Delta_{i}^{r}, 0 \leq i \leq r$, be the $i$-th face of the standard $r$-simplex $\Delta^{r}$. The restriction of a differentiable $r$-simplex $\sigma$ to $\Delta_{i}^{r}$ (given the suborbihedron structure, cf [3]) is a differentiable $(r-1)$-simplex, which will be denoted by $\sigma_{i}$. We define

$$
\partial \sigma=\sum_{i=0}^{r}(-1)^{i} \frac{\left|G_{i}\right|}{|G|} \sigma_{i}
$$

where $G_{i}, G$ are the groups assigned to the top cell of $\Delta_{i}^{r}, \Delta^{r}$ respectively. The boundary of a differentiable $r$-chain $c=\sum_{k} a_{k} \sigma_{k}$ is defined to be $\partial c=$ $\sum_{k} a_{k} \partial \sigma_{k}$, which clearly satisfies

$$
\partial \circ \partial=0 .
$$

Finally, the Stokes' theorem implies that for any differentiable $r$-chain $c$ and $(r-1)$-form $\alpha$,

$$
\int_{c} d \alpha=\int_{\partial c} \alpha
$$

For any orbifold $X$, let $\mathcal{H}_{*}(X), \mathcal{H}^{*}(X)$ be the homology and cohomology groups of differentiable chains (with $\mathbb{Z}$ coefficients) in $X$. There are canonical homomorphisms

$$
H_{d R}^{*}(X) \rightarrow \mathcal{H}^{*}(X) \otimes \mathbb{R}
$$

induced by integration over differentiable chains, and

$$
\mathcal{H}_{*}(X) \rightarrow H_{*}(X ; \mathbb{Q})
$$

which is defined at the chain level by

$$
\sigma \mapsto \frac{1}{|G|}|\sigma|
$$

for each differentiable $r$-simplex $\sigma: \Delta^{r} \rightarrow X$, where $|\sigma|$ is the induced singular $r$-simplex in the underlying space, and $|G|$ is the order of the group $G$ assigned to the top cell of $\Delta^{r}$.

Theorem 2.1 The canonical homomorphism $H_{d R}^{*}(X) \rightarrow \mathcal{H}^{*}(X) \otimes \mathbb{R}$ is isomorphic, and the canonical homomorphism $\mathcal{H}_{*}(X) \rightarrow H_{*}(X ; \mathbb{Q})$ is isomorphic over $\mathbb{Q}$. 
Theorem 2.1 will not be used in this paper, and its proof will be given elsewhere. But we remark that the key point in the proof is to show that $\mathcal{H}^{*}(X) \otimes \mathbb{Q}$ are the cohomology groups associated to a fine torsionless resolution of the constant sheaf $\mathbb{Q} \times X$, with which the proof follows by the usual sheaf theoretical argument, for instance, as in [20].

In light of Theorem 2.1, we will say that a differentiable cycle $c$ in $X$ (ie, a differentiable chain $c$ such that $\partial c=0)$ is Poincaré dual to a de Rham cohomology class $\gamma \in H_{d R}^{*}(X)$ if there is a closed form $\alpha \in \gamma$ such that for any closed form $\beta$ on $X$,

$$
\int_{c} \beta=\int_{X} \alpha \wedge \beta
$$

Here is a typical situation: Let $Y$ be a compact, closed, and oriented $r$ dimensional orbifold and $f: Y \rightarrow X$ be a differentiable map in the sense of 3]. Note that $Y$ can be triangulated such that with respect to the triangulation, $Y$ is natually an orbihedron (cf Part II of [3]). Thus the restriction of $f$ to each top simplex in the triangulation of $Y$ defines a differentiable $r$-simplex in $X$, and in this way $f(Y)$ naturally becomes a differentiable $r$-chain in $X$ which is a cycle because $Y$ is compact, closed, and oriented. Clearly, in this case we have

$$
\int_{f(Y)} \beta=\int_{Y} f^{*} \beta
$$

for any differential form $\beta$ on $X$.

\section{Adjunction and intersection formulae}

In this section, we derive the adjunction formula for pseudoholomorphic curves in an almost complex 4-orbifold and a corresponding formula which expresses the algebraic intersection number of two distinct pseudoholomorphic curves in terms of local contributions from their geometric intersection, extending relevant work of Gromov [7] and McDuff [14, 15] in the manifold setting.

First of all, some convention and terminology. In this section (and the previous one as well), the notion of orbifolds is more general in the sense that the group action on each uniformizing system needs not to be effective. The orbifolds in the classical sense where the group actions are effective are called reduced. The points which are the principal orbits in each uniformizing system are called regular points. They have the smallest isotropy groups in each connected component of the orbifold, which are all isomorphic, and they form an open, dense 
submanifold of the orbifold. The points in the complement of regular points are called orbifold points. When the orbifold is reduced and has no codimension 2 subsets of orbifold points, we also allow ourselves to use the usual terminologies, ie, "orbifold point" = "singular point" and "regular point" = "smooth point".

We now begin by setting the stage. Let $X$ be a compact, closed, and almost complex 4-dimensional orbifold which is canonically oriented by the almost complex structure $J$. We shall assume that the 4 -orbifold $X$ is reduced throughout. We shall also consider connected, compact, and closed complex orbifolds $\Sigma$ with $\operatorname{dim}_{\mathbb{C}} \Sigma=1$, namely the orbifold Riemann surfaces, which are not assumed to be reduced in general.

\section{Definition A}

A $J$-holomorphic curve in $X$ is a closed subset $C \subset X$ such that there is a nonconstant map $f: \Sigma \rightarrow X$ in the sense of [3] with $C=\operatorname{Im} f 2$ which obeys

(a) The representatives of $f$ are $J$-holomorphic.

(b) The homomorphisms between isotropy groups in each representative of $f$ are injective, and are isomorphic at all but at most finitely many regular points of $\Sigma$.

(c) The map $f$ is not multiply covered in the following sense: $f$ does not factor through any holomorphic map $\phi: \Sigma \rightarrow \Sigma^{\prime}$ to a map $f^{\prime}: \Sigma^{\prime} \rightarrow X$ such that the degree of the map induced by $\phi$ between the underlying Riemann surfaces is greater than one.

A $J$-holomorphic curve $C$ is called of type I if $\Sigma$ is reduced, and is called of type II otherwise. Clearly this definition is independent of the parametrization $f: \Sigma \rightarrow X$. Likewise, the order of the isotropy groups of the "regular" points in $C$, ie, the images of all but at most finitely many regular points in $\Sigma$ under $f$, depends only on $C$, and is called the multiplicity of $C$ and is denoted by $m_{C}$ throughout. A $J$-holomorphic curve $C$ is of type I if and only if $m_{C}=1$. A type I $J$-holomorphic curve is contained in the set of regular points of $X$ except for possibly finitely many points, and a type II $J$-holomorphic curve is contained entirely in the set of orbifold points of $X$. Finally, we remark that for a type I $J$-holomorphic curve $C$, any parametrization $f: \Sigma \rightarrow X$ of $C$ is uniquely determined by the induced map between the underlying spaces.

\footnotetext{
${ }^{2}$ Each map $f$ in the sense of $[3]$ induces a continuous map between the underlying spaces; by the image under such an $f$, we always mean the image under the map induced by $f$.
} 


\section{Definition B}

(1) For any $J$-holomorphic curve $C$ in $X$, the Poincaré dual of $C$ is defined to be the class $P D(C) \in H^{2}(X ; \mathbb{Q})$ which is uniquely determined by

$$
m_{C}^{-1} \alpha[C]=P D(C) \cup \alpha[X], \forall \alpha \in H^{2}(X ; \mathbb{Q}),
$$

where $[C]$ is the class of $C$ in $H_{2}(X ; \mathbb{Z})$.

(2) The algebraic intersection number of two $J$-holomorphic curves $C, C^{\prime}$ (not necessarily distinct) is defined to be

$$
C \cdot C^{\prime}=P D(C) \cup P D\left(C^{\prime}\right)[X] .
$$

We remark that the Poincaré dual $P D(C)$ differs from the usual one by a factor $m_{C}^{-1}$, thus is different for a type II $J$-holomorphic curve. On the other hand, if $C$ is parametrized by $f: \Sigma \rightarrow X$, the class of the differentiable cycle $f(\Sigma)$ in $\mathcal{H}_{2}(X)$ is sent to $m_{C}^{-1}[C]$ under the canonical homomorphism $\mathcal{H}_{2}(X) \rightarrow$ $H_{2}(X ; \mathbb{Q})$. In light of Theorem 2.1, $P D(C)$ is Poincaré dual to $f(\Sigma)$ under the canonical isomorphisms $H_{d R}^{2}(X) \cong \mathcal{H}^{2}(X) \otimes \mathbb{R} \cong H^{2}(X ; \mathbb{R})$.

We proceed further with a digression on some crucial local properties of $J$ holomorphic curves in $\mathbb{C}^{2}$ due to McDuff, cf [14, 15], where we assume that $\mathbb{C}^{2}$ is given with an almost complex structure $J$ which equals the standard structure at the origin. To fix the notation, the disc of radius $R$ in $\mathbb{C}$ centered at 0 is denoted by $D(R)$.

First, some local analytic properties of $J$-holomorphic curves:

- For any $J$-holomorphic curve $f:(D(R), 0) \rightarrow\left(\mathbb{C}^{2}, 0\right)$ where $f$ is not multiply covered, there exists an $0<R^{\prime} \leq R$ such that $\left.f\right|_{D\left(R^{\prime}\right) \backslash\{0\}}$ is embedded.

- Let $f:(D(R), 0) \rightarrow\left(\mathbb{C}^{2}, 0\right)$ be a $J$-holomorphic curve such that $\left.f\right|_{D(R) \backslash\{0\}}$ is embedded. Then for any sufficiently small $\epsilon>0$, there is an almost complex structure $J_{\epsilon}$ and a $J_{\epsilon}$-holomorphic immersion $f_{\epsilon}$ (not multiply covered) such that as $\epsilon \rightarrow 0, J_{\epsilon} \rightarrow J$ in $C^{1}$ topology and $f_{\epsilon} \rightarrow f$ in $C^{2}$ topology. Moreover, given any annuli $\{\lambda \leq|z| \leq R\}$ and $\left\{\lambda^{\prime} \leq|z| \leq \lambda\right\}$ in $D(R)$, one can arrange to have $f=f_{\epsilon}$ in $\{\lambda \leq|z| \leq R\}$ and to have $J_{\epsilon}=J$ except in a chosen neighborhood of the image of $\left\{\lambda^{\prime} \leq|z| \leq \lambda\right\}$ under $f$ by letting $\epsilon>0$ sufficiently small.

- Any two distinct $J$-holomorphic curves $f: D(R) \rightarrow \mathbb{C}^{2}, f^{\prime}: D\left(R^{\prime}\right) \rightarrow \mathbb{C}^{2}$ intersect at only finitely many points, ie, the set $\left\{\left(z, z^{\prime}\right) \in D(R) \times D\left(R^{\prime}\right) \mid\right.$ $\left.f(z)=f^{\prime}\left(z^{\prime}\right)\right\}$ is finite. 
Second, the local intersection and self-intersection number of $J$-holomorphic curves:

- Let $C, C^{\prime}$ be distinct $J$-holomorphic curves which are parametrized by $f:(D(R), 0) \rightarrow\left(\mathbb{C}^{2}, 0\right)$ and $f^{\prime}:\left(D\left(R^{\prime}\right), 0\right) \rightarrow\left(\mathbb{C}^{2}, 0\right)$, such that $\left.f\right|_{D(R) \backslash\{0\}}$ and $\left.f^{\prime}\right|_{D\left(R^{\prime}\right) \backslash\{0\}}$ are embedded and $0 \in \mathbb{C}^{2}$ is the only intersection of $C$ and $C^{\prime}$. Perturb $C$ into $\bar{C}$ (which may not be pseudoholomorphic), keeping $\partial C$ and $C$ disjoint from $C^{\prime}$ and $\partial C^{\prime}$ respectively, such that $\bar{C}$ intersects with $C^{\prime}$ transversely. Then the intersection number $C \cdot C^{\prime}$ is defined by counting the intersection of $\bar{C}$ and $C^{\prime}$ with signs. $C \cdot C^{\prime}$ may be determined using the following recipe: perturb $f, f^{\prime}$ into $J_{\epsilon}$-holomorphic immersions $f_{\epsilon}, f_{\epsilon}^{\prime}$, then

$$
C \cdot C^{\prime}=\sum_{\left\{\left(z, z^{\prime}\right) \mid f_{\epsilon}(z)=f_{\epsilon}^{\prime}\left(z^{\prime}\right)\right\}} t_{\left(z, z^{\prime}\right)}
$$

where $t_{\left(z, z^{\prime}\right)}=1$ when $f_{\epsilon}(z)=f_{\epsilon}^{\prime}\left(z^{\prime}\right)$ is a transverse intersection, and $t_{\left(z, z^{\prime}\right)}=n \geq 2$ when $f_{\epsilon}(z)=f_{\epsilon}^{\prime}\left(z^{\prime}\right)$ has tangency of order $n$. The intersection number $C \cdot C^{\prime}$ has the following properties: it depends only on the germs of $C, C^{\prime}$ at $0 \in \mathbb{C}^{2}$, it is always positive, and it equals one if and only if $C, C^{\prime}$ are both embedded and intersect at $0 \in \mathbb{C}^{2}$ transversely.

- Let $C$ be a $J$-holomorphic curve which is parametrized by $f:(D(R), 0)$ $\rightarrow\left(\mathbb{C}^{2}, 0\right)$ such that $\left.f\right|_{D(R) \backslash\{0\}}$ is embedded. Then the local self-intersection number $C \cdot C$ is well-defined, which can be determined using the following recipe: perturb $f$ into a $J_{\epsilon}$-holomorphic immersion $f_{\epsilon}$, then

$$
C \cdot C=\sum_{\left\{\left[z, z^{\prime}\right] \mid z \neq z^{\prime}, f_{\epsilon}(z)=f_{\epsilon}\left(z^{\prime}\right)\right\}} t_{\left[z, z^{\prime}\right]}
$$

where $\left[z, z^{\prime}\right]$ denotes the unordered pair of $z, z^{\prime}$, and where $t_{\left[z, z^{\prime}\right]}=1$ when $f_{\epsilon}(z)=f_{\epsilon}\left(z^{\prime}\right)$ is a transverse intersection, and $t_{\left[z, z^{\prime}\right]}=n \geq 2$ when $f_{\epsilon}(z)=f_{\epsilon}\left(z^{\prime}\right)$ has tangency of order $n$. The local self-intersection number $C \cdot C$ has the following properties: it depends only on the germ of $C$ at $0 \in \mathbb{C}^{2}$, and it is non-negative which equals zero if and only if $C$ is embedded.

End of digression.

In order to state the adjunction and intersection formulae, we need to further introduce some definitions.

(1) Recall from [3] that a representative of a map $f: \Sigma \rightarrow X$ parametrizing a $J$-holomorphic curve $C$ gives rise to a collection of pairs $\left(f_{i}, \rho_{i}\right):\left(\widehat{D_{i}}, G_{D_{i}}\right) \rightarrow$ $\left(\widehat{U}_{i}, G_{U_{i}}\right)$ satisfying certain compatibility conditions, where $\left\{\left(\widehat{D_{i}}, G_{D_{i}}\right)\right\}$, 
$\left\{\left(\widehat{U}_{i}, G_{U_{i}}\right)\right\}$ are a collection of uniformizing systems of $\Sigma$ and $X$ respectively, and each $\rho_{i}$ is a homomorphism, which is injective by (b) of Definition A, and each $f_{i}$ is a $\rho_{i}$-equivariant $J$-holomorphic map. We may assume without loss of generality that each $\widehat{D_{i}}$ is a disc centered at $0 \in \mathbb{C}$ and each $\widehat{U}_{i}$ is a ball centered at $0 \in \mathbb{C}^{2}$, and $G_{D_{i}}, G_{U_{i}}$ act linearly. Moreover, because of (b) and (c) in Definition A, we may assume that each $f_{i}$ is embedded when restricted to $\widehat{D_{i}} \backslash\{0\}$ and $\rho_{i}\left(G_{D_{i}}\right)$ is the subgroup of $G_{U_{i}}$ which leaves $f_{i}\left(\widehat{D_{i}}\right) \subset \widehat{U}_{i}$ invariant. (The case of type II is explained in the proof of Lemma 3.4 below.) Let $z$ be the orbit of $0 \in \widehat{D_{i}}$ in $\Sigma$. We shall call the germ of $\operatorname{Im} f_{i}$ at $0 \in \widehat{D_{i}}$ a local representative of the $J$-holomorphic curve $C=\operatorname{Im} f$ at $z \in \Sigma$. The set $\Lambda(C)_{z}$ of all local representatives of $C$ at $z$ is clearly the set of germs of the elements in

$$
\left\{\operatorname{Im}\left(g \circ f_{i}\right) \mid g \in G_{U_{i}}\right\},
$$

which is naturally parametrized by the coset $G_{U_{i}} / \rho_{i}\left(G_{D_{i}}\right)$. Note that for all but at most finitely many points $z \in \Sigma$, the set $\Lambda(C)_{z}$ of local representatives of $C$ at $z$ contains only one element.

(2) For any $J$-holomorphic curve $C$ in $X$, its virtual genus is defined to be

$$
g(C)=\frac{1}{2}(C \cdot C+c(C))+\frac{1}{m_{C}}
$$

where $c=-c_{1}(T X)$. Note that $g(C)$ is a rational number in general.

(3) Let $\Sigma$ be an (connected) orbifold Riemann surface, and let $m_{\Sigma}$ be the order (of isotropy groups) of its regular points and $m_{1}, m_{2}, \cdots, m_{k}$ be the orders (of isotropy groups) of its orbifold points. We define the orbifold genus of $\Sigma$ by

$$
g_{\Sigma}=\frac{g_{|\Sigma|}}{m_{\Sigma}}+\sum_{i=1}^{k}\left(\frac{1}{2 m_{\Sigma}}-\frac{1}{2 m_{i}}\right),
$$

where $g_{|\Sigma|}$ is the genus of the underlying Riemann surface of $\Sigma$. Note that with the above definition, $c_{1}(T \Sigma)(\Sigma)=2 m_{\Sigma}^{-1}-2 g_{\Sigma}$ where $T \Sigma$ is the orbifold tangent bundle.

With the preceding understood, consider the following:

Theorem 3.1 (Adjunction Formula) Let $C$ be a $J$-holomorphic curve which is parametrized by $f: \Sigma \rightarrow X$. Then

$$
g(C)=g_{\Sigma}+\sum_{\left\{\left[z, z^{\prime}\right] \mid z \neq z^{\prime}, f(z)=f\left(z^{\prime}\right)\right\}} k_{\left[z, z^{\prime}\right]}+\sum_{z \in \Sigma} k_{z},
$$

where $\left[z, z^{\prime}\right]$ denotes the unordered pair of $z, z^{\prime}$, and where the numbers $k_{\left[z, z^{\prime}\right]}, k_{z}$ are defined as follows. 
- Let $G_{\left[z, z^{\prime}\right]}$ be the isotropy group at $f(z)=f\left(z^{\prime}\right)$ and $\Lambda(C)_{z}=\left\{C_{z, \alpha}\right\}$, $\Lambda(C)_{z^{\prime}}=\left\{C_{z^{\prime}, \alpha^{\prime}}\right\}$, then

$$
k_{\left[z, z^{\prime}\right]}=\frac{1}{\left|G_{\left[z, z^{\prime}\right]}\right|} \sum_{\alpha, \alpha^{\prime}} C_{z, \alpha} \cdot C_{z^{\prime}, \alpha^{\prime}} .
$$

- Let $G_{z}$ be the isotropy group at $f(z)$ and $\Lambda(C)_{z}=\left\{C_{z, \alpha}\right\}$, then

$$
k_{z}=\frac{1}{2\left|G_{z}\right|}\left(\sum_{\alpha} C_{z, \alpha} \cdot C_{z, \alpha}+\sum_{\alpha, \beta} C_{z, \alpha} \cdot C_{z, \beta}\right) .
$$

(Note: the second sum is over all $\alpha, \beta$ which are not necessarily distinct.)

Theorem 3.2 (Intersection Formula) Let $C, C^{\prime}$ be distinct $J$-holomorphic curves parametrized by $f: \Sigma \rightarrow X, f^{\prime}: \Sigma^{\prime} \rightarrow X$ respectively. Then the algebraic intersection number

$$
C \cdot C^{\prime}=\sum_{\left\{\left(z, z^{\prime}\right) \mid f(z)=f^{\prime}\left(z^{\prime}\right)\right\}} k_{\left(z, z^{\prime}\right)}
$$

where $k_{\left(z, z^{\prime}\right)}$ is defined as follows. Let $G_{\left(z, z^{\prime}\right)}$ be the isotropy group at $f(z)=$ $f^{\prime}\left(z^{\prime}\right)$ and $\Lambda(C)_{z}=\left\{C_{z, \alpha}\right\}, \Lambda\left(C^{\prime}\right)_{z^{\prime}}=\left\{C_{z^{\prime}, \alpha^{\prime}}^{\prime}\right\}$, then

$$
k_{\left(z, z^{\prime}\right)}=\frac{1}{\left|G_{\left(z, z^{\prime}\right)}\right|} \sum_{\alpha, \alpha^{\prime}} C_{z, \alpha} \cdot C_{z^{\prime}, \alpha^{\prime}}^{\prime}
$$

The adjunction formula implies the following:

Corollary 3.3 Let $C$ be a $J$-holomorphic curve parametrized by $f: \Sigma \rightarrow X$. Then the virtual genus of $C$ is greater than or equal to the orbifold genus of $\Sigma$, ie, $g(C) \geq g_{\Sigma}$, with $g(C)=g_{\Sigma}$ iff $C$ is a suborbifold of $X$ and $f$ is an orbifold embedding.

The rest of this section is occupied by the proof of Theorem 3.1 and Theorem 3.2. We begin with some preliminary lemmas.

Lemma 3.4 Let $C$ be a type II $J$-holomorphic curve parametrized by $f: \Sigma \rightarrow$ $X$. Then $f$ is represented by a collection of pairs $\left\{\left(f_{i}, \rho_{i}\right)\right\}$ where each $f_{i}$ is an embedding.

Proof Let $\left(\widehat{U}, G_{U}\right)$ be a uniformizing system of $X$, where $\widehat{U}$ is a ball in $\mathbb{C}^{2}$ and $G_{U}$ is nontrivial and acts linearly. We say that $G_{U}$ is of type A if the 
fixed-point set of $G_{U}$ is a complex line in $\mathbb{C}^{2}$, and that $G_{U}$ is of type B if $0 \in \mathbb{C}^{2}$ is the only fixed point.

Let $\left\{\left(f_{i}, \rho_{i}\right)\right\}$ be a representative of $f(\operatorname{cf}[3])$, where each $\left(f_{i}, \rho_{i}\right):\left(\widehat{D_{i}}, G_{D_{i}}\right) \rightarrow$ $\left(\widehat{U}_{i}, G_{U_{i}}\right)$. Since $C$ is of type II, each $G_{U_{i}}$ is nontrivial. Consider the case where $G_{U_{i}}$ is of type A first. In this case, $\operatorname{Im} f_{i}$ lies in the complex line which is fixed by $G_{U_{i}}$, therefore $f_{i}$ is a holomorphic map between two discs in $\mathbb{C}$. It follows that $f_{i}$ is either an embedding or a branched covering. Suppose $f_{i}$ is a branched covering, and without loss of generality assume that $0 \in \widehat{D_{i}}$ is the only branching point. Then there are $z, z^{\prime} \neq 0$ in $\widehat{D_{i}}$ with $z \neq z^{\prime}$, such that $f_{i}(z)=f_{i}\left(z^{\prime}\right) \in \widehat{U}_{i}$. Since $f$ is not multiply covered, there must be a $g \in G_{D_{i}}$ such that $g \cdot z=z^{\prime}$. On the other hand, by (b) of Definition A, $\rho_{i}$ is an isomorphism onto $G_{U_{i}}$ when restricted to the isotropy subgroup of $z$, so that there is an $h \in G_{D_{i}}$ fixing $z$ such that $\rho_{i}(h)=\rho_{i}(g)$. It is easily seen that $\rho_{i}\left(g h^{-1}\right)=1 \in G_{U_{i}}$ but $g h^{-1} \neq 1 \in G_{D_{i}}$, a contradiction to the assumption in (b) of Definition A that $\rho_{i}$ is injective. Hence $f_{i}$ is an embedding. When $G_{U_{i}}$ is of type B, $\operatorname{Im} f_{i}$ lies in a complex line in $\mathbb{C}^{2}$ whose isotropy is a proper subgroup $H$ of $G_{U_{i}}$. Again $f_{i}$ is either an embedding or a branched covering. If $f_{i}$ is a branched covering, then there are $z, z^{\prime} \neq 0$ in $\widehat{D_{i}}$ with $z \neq z^{\prime}$, such that $f_{i}(z)=f_{i}\left(z^{\prime}\right) \in \widehat{U}_{i}$. Moreover, since $f$ is not multiply covered, there is a $g \in G_{D_{i}}$ such that $g \cdot z=z^{\prime}$, and in this case, note that $\rho_{i}(g) \in H$. On the other hand, there is an $h$ in the isotropy subgroup of $z$ such that $\rho_{i}(h)=\rho_{i}(g) \in H$, which gives a contradiction as in the type A case. Hence the lemma.

Lemma 3.5 Let $C$ be a $J$-holomorphic curve parametrized by $f: \Sigma \rightarrow X$. Then there is a closed 2 -form $\eta_{C}$ on $X$ which represents the Poincaré dual of the differentiable cycle $f(\Sigma)$ in $X$, ie, for any 2 -form $\alpha$ on $X$,

$$
\int_{\Sigma} f^{*} \alpha=\int_{X} \eta_{C} \wedge \alpha
$$

Moreover, $\eta_{C}$ may be chosen such that it is supported in any given neighborhood of $C$ in $X$.

Proof We consider the case where $C$ is of type I first.

To fix the notation, let $z_{1}, z_{2}, \cdots, z_{k}$ be the set of points in $\Sigma$ whose image under $f$ is an orbifold point in $X$. For each $i=1,2, \cdots, k$, we set $p_{i}=f\left(z_{i}\right)$ and let $m_{i} \geq 1$ be the order of the isotropy group at $z_{i}$. Furthermore, we denote by $\left(\widehat{D}_{i}, \mathbb{Z}_{m_{i}}\right),\left(\widehat{V}_{i}, G_{i}\right)$ some local uniformizing systems at $z_{i}, p_{i}$ respectively, and denote by $\left(f_{i}, \rho_{i}\right):\left(\widehat{D_{i}}, \mathbb{Z}_{m_{i}}\right) \rightarrow\left(\widehat{V}_{i}, G_{i}\right)$ a local representative of $f$ at $z_{i}$ such that $f_{i}$ is embedded when restricted to $\widehat{D_{i}} \backslash\{0\}$. Set $D_{i}=\widehat{D_{i}} / \mathbb{Z}_{m_{i}}$ 
and $V_{i}=\widehat{V}_{i} / G_{i}$ for the corresponding neighborhood of $z_{i}$ and $p_{i}$ in $\Sigma$ and $X$ respectively. Without loss of generality, we may assume that $D_{i}$ is the connected component of $f^{-1}\left(V_{i}\right)$ that contains $z_{i}$.

For each critical point $z$ of $f$ (ie $d f(z)=0$ ) where $f(z)$ is a regular point in $X$, we perturb $f$ locally in a small neighborhood of $z$ into a $J_{\epsilon}$-holomorphic immersion, which is supported in the complement of $\bigcup_{i=1}^{k} D_{i}$, and for each $i=1,2, \cdots, k$, we perturb $f_{i}$ into a $J_{\epsilon}$-holomorphic immersion $f_{i, \epsilon}$ (if $f_{i}$ is already embedded, we simply let $f_{i, \epsilon}=f_{i}$ ). Let $\widehat{D_{i}^{\prime}} \subset \widehat{D_{i}}$ be a closed disc of a smaller radius such that $f_{i, \epsilon}=f_{i}$ over $\widehat{D_{i}} \backslash \widehat{D_{i}^{\prime}}$. We set $\Sigma_{0}=\Sigma \backslash \bigcup_{i=1}^{k} D_{i}$ and $\Sigma_{0}^{\prime}=\Sigma \backslash \bigcup_{i=1}^{k} D_{i}^{\prime}$ where $D_{i}^{\prime}=\widehat{D_{i}^{\prime}} / \mathbb{Z}_{m_{i}}$, and we denote the perturbation of $f$ over $\Sigma_{0}^{\prime}$ by $f_{\epsilon}$, which is a $J_{\epsilon}$-holomorphic immersion into $X^{0}$, the complement of orbifold points in $X$. Note that $f_{i, \epsilon}$ may not be $\rho_{i}$-equivariant, and $J_{\epsilon}$ may not be $G_{i}$-equivariant over $\widehat{V}_{i}$. Hence $f_{\epsilon}, f_{i, \epsilon}, i=1,2, \cdots, k$, may not define a pseudoholomorphic curve in $X$. Nevertheless, for any closed 2-form $\alpha$ on $X$, it is easily seen that

$$
\int_{\Sigma} f^{*} \alpha=\int_{\Sigma_{0}} f_{\epsilon}^{*} \alpha+\sum_{i=1}^{k} \frac{1}{m_{i}} \int_{\widehat{D}_{i}} f_{i, \epsilon}^{*} \alpha
$$

Let $\nu_{\epsilon}=f_{\epsilon}^{*} T X^{0} / T \Sigma_{0}^{\prime}$ be the normal bundle of the immersion $f_{\epsilon}$ in $X^{0}$, and let $\nu_{i, \epsilon}=f_{i, \epsilon}^{*} T \widehat{V}_{i} / T \widehat{D}_{i}$ be the normal bundle of the immersion $f_{i, \epsilon}$ in $\widehat{V}_{i}, i=$ $1,2, \cdots, k$. We fix an immersion $\bar{f}_{\epsilon}$ of a tubular neighborhood of the zero section of $\nu_{\epsilon}$ into $X^{0}$, and fix an immersion $\bar{f}_{i, \epsilon}$ of a tubular neighborhood of the zero section of $\nu_{i, \epsilon}$ into $\widehat{V}_{i}$ for each $i$, which are assumed to be compatible on the overlaps. We denote by $\overline{\Theta_{\epsilon}}, \overline{\Theta_{i, \epsilon}}$ the push-forward of some Thom forms $\Theta_{\epsilon}, \Theta_{i, \epsilon}$ of $\nu_{\epsilon}, \nu_{i, \epsilon}$ by $\bar{f}_{\epsilon}, \bar{f}_{i, \epsilon}$ respectively, where $\Theta_{\epsilon}, \Theta_{i, \epsilon}$ are compatible on the overlaps. Finally, let $x_{1}, x_{2}, \cdots, x_{l}$ be the set $\left\{p_{i} \mid i=1,2, \cdots, k\right\}$. For each $x_{j}, j=1,2, \cdots, l$, let $\left(\widehat{V_{x_{j}}}, G_{x_{j}}\right)$ be a local uniformizing system at $x_{j}$. Without loss of generality, we assume $V_{i}=V_{x_{j}}=\widehat{V_{x_{j}}} / G_{x_{j}}$ whenever $p_{i}=x_{j}$.

With the preceding understood, the 2 -form $\eta_{C}$ is defined as follows. On $X \backslash$ $\bigcup_{j=1}^{l} V_{x_{j}}, \eta_{C}=\overline{\Theta_{\epsilon}}$, and on each $\widehat{V_{x_{j}}}, j=1,2, \cdots, l$,

$$
\eta_{C}=\sum_{\left\{i \mid p_{i}=x_{j}\right\}} \frac{1}{m_{i}} \sum_{g \in G_{x_{j}}} g^{*} \overline{\Theta_{i, \epsilon}} .
$$


Now for any 2-form $\alpha$ on $X$, we have

$$
\begin{aligned}
\int_{X} \eta_{C} \wedge \alpha & =\int_{X \backslash \bigcup_{j=1}^{l} V_{x_{j}}} \eta_{C} \wedge \alpha+\sum_{j=1}^{l} \frac{1}{\left|G_{x_{j}}\right|} \int_{\widehat{V_{x_{j}}}} \eta_{C} \wedge \alpha \\
& =\int_{X \backslash \bigcup_{j=1}^{l} V_{x_{j}}} \overline{\Theta_{\epsilon}} \wedge \alpha+\sum_{j=1}^{l} \frac{1}{\left|G_{x_{j}}\right|} \int_{\widehat{V_{x_{j}}}}\left(\sum_{\left\{i \mid p_{i}=x_{j}\right\}} \frac{1}{m_{i}} \sum_{g \in G_{x_{j}}} g^{*} \overline{\Theta_{i, \epsilon}}\right) \wedge \alpha \\
& =\int_{\Sigma_{0}} f_{\epsilon}^{*} \alpha+\sum_{j=1}^{l} \sum_{\left\{i \mid p_{i}=x_{j}\right\}} \frac{1}{m_{i}}\left(\frac{1}{\left|G_{x_{j}}\right|} \int_{\widehat{V_{x_{j}}}} \sum_{g \in G_{x_{j}}} g^{*}\left(\overline{\Theta_{i, \epsilon}} \wedge \alpha\right)\right) \\
& =\int_{\Sigma_{0}} f_{\epsilon}^{*} \alpha+\sum_{j=1}^{l} \sum_{\left\{i \mid p_{i}=x_{j}\right\}} \frac{1}{m_{i}} \int_{\widehat{V_{x_{j}}}} \overline{\Theta_{i, \epsilon}} \wedge \alpha \\
& =\int_{\Sigma_{0}} f_{\epsilon}^{*} \alpha+\sum_{i=1}^{k} \frac{1}{m_{i}} \int_{\widehat{D_{i}}} f_{i, \epsilon}^{*} \alpha=\int_{\Sigma} f^{*} \alpha .
\end{aligned}
$$

Hence $\eta_{C}$ represents the Poincaré dual of the differentiable cycle $f(\Sigma)$. By way of construction, $\eta_{C}$ may be chosen to be supported in any given neighborhood of $C$ in $X$.

Next we consider the case where $C$ is of type II.

By Lemma 3.4, $\nu=f^{*} T X / T \Sigma$ is an orbifold complex line bundle over $\Sigma$. Let $\Theta$ be a Thom form of $\nu$. Then notice that $\nu$ is sort of a quasi-normal bundle of $C$ in $X$ in the sense that one can push-forward $\Theta$ to $X$. The resulting form, which is defined to be $\eta_{C}$, is a closed 2-form on $X$, supported in any given neighborhood of $C$, and for any $x \in C$, there exists a local uniformizing system $(\widehat{V}, G)$ at $x$ such that on $\widehat{V}$,

$$
\eta_{C}=\sum_{i=1}^{l} \frac{1}{m_{i}} \sum_{g \in G} g^{*} \overline{\Theta_{i}},
$$

where $f^{-1}(x)=\left\{z_{1}, z_{2}, \cdots, z_{l}\right\}, m_{i}$ is the order of $z_{i}$ in $\Sigma$, and $\overline{\Theta_{i}}$ is the pushforward of $\Theta$ to $V$ associated to some arbitrarily fixed choice of representatives of the parametrization $f: \Sigma \rightarrow X$ of $C$. As in the case where $C$ is of type I, we have for any 2 -form $\alpha$ on $X$

$$
\int_{X} \eta_{C} \wedge \alpha=\int_{\Sigma} f^{*} \alpha
$$

so that $\eta_{C}$ represents the Poincaré dual of the differentiable cycle $f(\Sigma)$. 
Note that by the above lemma, we have

$$
C \cdot C^{\prime}=\int_{X} \eta_{C} \wedge \eta_{C^{\prime}}
$$

for the algebraic intersection number of two $J$-holomorphic curves $C, C^{\prime}$.

The next lemma is concerned with a formula which expresses the first Chern class of an orbifold complex vector bundle over a reduced orbifold Riemann surface in terms of the first Chern class over the complement of the orbifold points with respect to a certain canonical trivialization and the "first Chern class" at each orbifold point. To be more precise, let $E \rightarrow \Sigma$ be a rank $n$ orbifold complex vector bundle over a reduced orbifold Riemann surface. Let $z_{1}, z_{2}, \cdots, z_{k} \in \Sigma$ be any given set of points which contains the set of orbifold points, and let $m_{1}, m_{2}, \cdots, m_{k}$ be the orders of the corresponding isotropy groups. Suppose over a local uniformizing system $\left(\widehat{D_{i}}, \mathbb{Z}_{m_{i}}\right)$ at each $z_{i}$, the orbifold bundle $E$ has a trivialization $\left(\widehat{D_{i}} \times \mathbb{C}^{n}, \mathbb{Z}_{m_{i}}\right)$, such that $\mathbb{Z}_{m_{i}}$ acts on $\widehat{D_{i}} \times \mathbb{C}^{n}$ by

$$
\mu_{m_{i}} \cdot\left(z, v_{1}, v_{2}, \cdots, v_{n}\right)=\left(\mu_{m_{i}} z, \mu_{m_{i}}^{m_{i, 1}} v_{1}, \mu_{m_{i}}^{m_{i, 2}} v_{2}, \cdots, \mu_{m_{i}}^{m_{i, n}} v_{n}\right),
$$

where $\mu_{m_{i}}=\exp \left(\sqrt{-1} \frac{2 \pi}{m_{i}}\right)$ is the generator of $\mathbb{Z}_{m_{i}}$, and $0 \leq m_{i, j}<m_{i}, j=$ $1,2, \cdots, n$. Set $D_{i}=\widehat{D_{i}} / \mathbb{Z}_{m_{i}}, \Sigma_{0}=\Sigma \backslash \bigcup_{i=1}^{k} D_{i}$, and $E_{0}=\left.E\right|_{\Sigma_{0}}$. We consider the trivialization $\tau$ of $E_{0}$ over $\partial \Sigma_{0}=\bigcup_{i=1}^{k} \partial D_{i}$ where along each $\partial D_{i}, \tau$ is given by pushing down a set of equivariant sections $\left\{s_{j}(z) \mid j=1,2, \cdots, n\right\}$ of $\partial \widehat{D_{i}} \times \mathbb{C}^{n}$ over $\partial \widehat{D_{i}}$, where $s_{j}(z)=\left(0, \cdots, z^{m_{i, j}}, \cdots, 0\right), j=1,2, \cdots, n$. Let $\partial D_{i} \times \mathbb{C}^{n}$ be the trivialization $\tau$ of $E_{0}$ over $\partial D_{i}$. Then the canonical map $\psi_{i}: \partial \widehat{D_{i}} \times \mathbb{C}^{n} \rightarrow \partial D_{i} \times \mathbb{C}^{n}$ is given by

$$
\psi_{i}\left(z, v_{1}, v_{2}, \cdots, v_{n}\right)=\left(z^{m_{i}}, z^{-m_{i, 1}} v_{1}, z^{-m_{i, 2}} v_{2}, \cdots, z^{-m_{i, n}} v_{n}\right) .
$$

With the preceding understood, the said formula is the following:

Lemma 3.6 $c_{1}(E)(\Sigma)=c_{1}\left(E_{0}, \tau\right)\left(\Sigma_{0}, \partial \Sigma_{0}\right)+\sum_{i=1}^{k}\left(\sum_{j=1}^{n} \frac{m_{i, j}}{m_{i}}\right)$.

Proof Let $\nabla_{0}$ be a unitary connection of $E_{0}$ which is trivial with respect to the trivialization $\tau$ along the boundary $\partial \Sigma_{0}$. Over each $\left(\widehat{D_{i}} \times \mathbb{C}^{n}, \mathbb{Z}_{m_{i}}\right)$, we define an equivariant connection $\nabla=\beta \psi_{i}^{*} \nabla_{0}+(1-\beta) d$ where $\beta$ is an equivariant cutoff function equaling one near $\partial \widehat{D_{i}}$ and $d$ is the trivial connection with respect to the natural trivialization of $\widehat{D_{i}} \times \mathbb{C}^{n}$. Clearly $\nabla_{0}, \nabla$ are compatible on the overlaps so that they define a connection of the orbifold bundle $E$, which is still denoted by $\nabla$ for simplicity. We observe that over $\Sigma_{0}, \nabla=\nabla_{0}$, and with respect to each local trivialization $\left(\widehat{D_{i}} \times \mathbb{C}^{n}, \mathbb{Z}_{m_{i}}\right)$, the curvature form $F(\nabla)$ is 
given by the diagonal matrix whose entries are $-d\left(\beta m_{i, 1} \frac{d z}{z}\right), \cdots,-d\left(\beta m_{i, n} \frac{d z}{z}\right)$. Hence

$$
\begin{aligned}
c_{1}(E)(\Sigma) & =\int_{\Sigma} \frac{\sqrt{-1}}{2 \pi} \operatorname{tr} F(\nabla) \\
& =\int_{\Sigma_{0}} \frac{\sqrt{-1}}{2 \pi} \operatorname{tr} F\left(\nabla_{0}\right)+\sum_{i=1}^{k} \frac{1}{m_{i}} \int_{\widehat{D_{i}}} \frac{\sqrt{-1}}{2 \pi} \operatorname{tr} F(\nabla) \\
& =c_{1}\left(E_{0}, \tau\right)\left(\Sigma_{0}, \partial \Sigma_{0}\right)+\sum_{i=1}^{k}\left(\sum_{j=1}^{n} \frac{m_{i, j}}{m_{i}}\right) .
\end{aligned}
$$

As an example which is also relevant in the later discussion, we consider the case where $E=T \Sigma \rightarrow \Sigma$. On each local uniformizing system $\left(\widehat{D_{i}}, \mathbb{Z}_{m_{i}}\right), T \Sigma$ has a natural trivialization $\left(\widehat{D_{i}} \times \mathbb{C}, \mathbb{Z}_{m_{i}}\right)$ defined by the section $\frac{\partial}{\partial z}$, where $\mathbb{Z}_{m_{i}}$ acts by complex multiplication (ie $m_{i, 1}=1$ ). On the other hand, the trivialization $\tau$ is defined by $d \phi_{i}\left(z \frac{\partial}{\partial z}\right)=m_{i} w \frac{\partial}{\partial w}$ along each $\partial D_{i}$, where $\phi_{i}: \widehat{D_{i}} \rightarrow D_{i}$ is the map $w=z^{m_{i}}$. It is easily seen that $c_{1}\left(T \Sigma_{0}, \tau\right)\left(\Sigma_{0}, \partial \Sigma_{0}\right)=2-2 g_{|\Sigma|}-k$ where $g_{|\Sigma|}$ is the genus of the underlying Riemann surface of $\Sigma$, and $k$ is the number of components in $\partial \Sigma_{0}$. Hence Lemma 3.6 recovers the formula

$$
c_{1}(T \Sigma)(\Sigma)=2-2 g_{|\Sigma|}-\sum_{i=1}^{k}\left(1-\frac{1}{m_{i}}\right) .
$$

Note that the right hand side of the above equation equals $2-2 g_{\Sigma}$ by the definition of the orbifold genus $g_{\Sigma}$.

\section{Proof of Theorem 3.1}

We consider first the case where $C$ is a type I $J$-holomorphic curve. We shall continue to use the notations introduced in the proof of Lemma 3.5.

Let $E \rightarrow \Sigma$ be the pullback of $T X$ by $f$, which is a rank 2 orbifold complex vector bundle. Over each local uniformizing system $\left(\widehat{D_{i}}, \mathbb{Z}_{m_{i}}\right), E$ has a trivialization $\left(\widehat{D_{i}} \times \mathbb{C}^{2}, \mathbb{Z}_{m_{i}}\right)$, where $\{z\} \times \mathbb{C}^{2}, \forall z \in \widehat{D_{i}}$, is identified with $\left.T \widehat{V}_{i}\right|_{f_{i}(z)}$, and $\mathbb{Z}_{m_{i}}$ acts by $\mu_{m_{i}} \cdot(z, w)=\left(\mu_{m_{i}} z, \rho_{i}\left(\mu_{m_{i}}\right)(w)\right), \mu_{m_{i}}=\exp \left(\sqrt{-1} \frac{2 \pi}{m_{i}}\right)$. More concretely, we may identify $\widehat{V}_{i}$ with $\mathbb{C}^{2}$ such that the almost complex structure $J$ equals the standard one at the origin 0 , and there are coordinates $u, v$ such that $\rho_{i}\left(\mu_{m_{i}}\right)$ acts linearly as a diagonal matrix, say with entries $\mu_{m_{i}}^{m_{i, 1}}$, $\mu_{m_{i}}^{m_{i, 2}}$ where $0 \leq m_{i, 1}, m_{i, 2}<m_{i}$, and that $f_{i}(z)=\left(z^{l_{i}}, a_{i} z^{l_{i}}\right)+O\left(|z|^{l_{i}+1}\right)$ 
for some integer $l_{i} \geq 1$ and $a_{i} \in \mathbb{C}$. Observe that if $a_{i} \neq 0$, then $f_{i}$ being $\rho_{i}$-equivariant implies that $m_{i, 1}=m_{i, 2}$, so that we may modify with a linear coordinate change $(u, v) \mapsto\left(u, v-a_{i} u\right)$ such that $\rho_{i}\left(\mu_{m_{i}}\right)$ is still diagonalized and $f_{i}(z)=\left(z^{l_{i}}, 0\right)+O\left(|z|^{l_{i}+1}\right)$. Thus in any event, we have $f_{i}(z)=\left(z^{l_{i}}, 0\right)+O\left(|z|^{l_{i}+1}\right)$. Let $E_{0}=\left.E\right|_{\Sigma_{0}}$, and $\tau$ be the canonical trivialization of $E_{0}$ along $\partial \Sigma_{0}$ which is determined by the equivariant sections $\left(z^{\left.m_{i, 1}, 0\right)}\right.$ and $\left(0, z^{m_{i, 2}}\right)$ of $\widehat{D_{i}} \times \mathbb{C}^{2} \rightarrow \widehat{D_{i}}$ along each $\partial D_{i}$. Recall that $c=-c_{1}(T X)$. Hence by Lemma 3.6,

$$
c(C)=-c_{1}\left(E_{0}, \tau\right)\left(\Sigma_{0}, \partial \Sigma_{0}\right)-\sum_{i=1}^{k} \frac{m_{i, 1}+m_{i, 2}}{m_{i}} .
$$

Observe that $f_{\epsilon}^{*} T X^{0}=E_{0}$ along $\partial \Sigma_{0} \subset \Sigma_{0}^{\prime}$. Hence the canonical trivialization $\tau$ of $E_{0}$ along $\partial \Sigma_{0}$ gives rise to a trivialization of $f_{\epsilon}^{*} T X^{0}$ along $\partial \Sigma_{0}^{\prime}$, which is also denoted by $\tau$ for simplicity. Furthermore, note that $c_{1}\left(E_{0}, \tau\right)\left(\Sigma_{0}, \partial \Sigma_{0}\right)=$ $c_{1}\left(f_{\epsilon}^{*} T X^{0}, \tau\right)\left(\Sigma_{0}^{\prime}, \partial \Sigma_{0}^{\prime}\right)$. On the other hand, let $\tau_{h}$ be the trivialization of $T \Sigma_{0}^{\prime}$ along the boundary $\partial \Sigma_{0}^{\prime}$ given by the section $w \frac{\partial}{\partial w}$ (here $w$ is the holomorphic coordinate of each $D_{i}$ ). Then $\tau, \tau_{h}$ determine a unique trivialization $\tau_{v}$ of $\nu_{\epsilon}$ along $\partial \Sigma_{0}^{\prime}$ such that

$$
c_{1}\left(f_{\epsilon}^{*} T X^{0}, \tau\right)=c_{1}\left(T \Sigma_{0}^{\prime}, \tau_{h}\right)+c_{1}\left(\nu_{\epsilon}, \tau_{v}\right) .
$$

There are canonical bundle morphisms $\left.\left.\nu_{i, \epsilon}\right|_{\partial \widehat{D_{i}}} \rightarrow \nu_{\epsilon}\right|_{\partial D_{i}}$ induced by $\phi_{i}: \widehat{D_{i}} \rightarrow$ $D_{i}$ where $\phi_{i}(z)=z^{m_{i}}$. Through these bundle morphisms, the trivialization $\tau_{v}$ gives rise to a trivialization $\tau_{i, v}$ of $\nu_{i, \epsilon}$ along $\partial \widehat{D_{i}}$. In order to determine $\tau_{i, v}$, we recall that $f_{i}(z)=\left(z^{l_{i}}, 0\right)+O\left(|z|^{l_{i}+1}\right)$ and $f_{i, \epsilon}=f_{i}$ in $\widehat{D_{i}} \backslash \widehat{D_{i}^{\prime}}$. If we let $\tau_{i, h}$ be the trivialization of $T \widehat{D_{i}}$ along $\partial \widehat{D_{i}}$ (as a sub-bundle of $f_{i, \epsilon}^{*} T \widehat{V}_{i}$ ) which is induced by the trivialization $\tau_{h}$ of $T \Sigma_{0}^{\prime}$ along $\partial \Sigma_{0}^{\prime}$ through $\phi_{i}$, then $\tau_{i, h}$ is given by the section $\left(l_{i} z^{l_{i}}, 0\right)$ up to homotopy. Hence $\tau_{i, v}$ is given by the section $\left(0, z^{-l_{i}+m_{i, 1}+m_{i, 2}}\right)$ up to homotopy, since $\tau$ is given by the sections $\left(z^{m_{i, 1}, 0}\right)$ and $\left(0, z^{m_{i, 2}}\right)$.

We push $f_{\epsilon}$ off near $\partial \Sigma_{0}^{\prime}$ along the direction given by the trivialization $\tau_{v}$ of the normal bundle $\nu_{\epsilon}$ (note that $f_{\epsilon}$ is embedded near $\partial \Sigma_{0}^{\prime}$ ). Call the resulting map $f_{\epsilon}^{\prime}$. Correspondingly, each $f_{i, \epsilon}$ is pushed off near $\partial \widehat{D_{i}}$ to a $f_{i, \epsilon}^{\prime}$ along the direction given by the trivialization $\tau_{i, v}$ of the normal bundle $\nu_{i, \epsilon}$. As in the proof of Lemma 3.5, we can similarly construct a closed 2-form $\eta_{C}^{\prime}$ using $f_{\epsilon}^{\prime}, f_{i, \epsilon}^{\prime}$ instead of $f_{\epsilon}, f_{i, \epsilon}$, which is also Poincaré dual to the differentiable cycle $f(\Sigma)$. Furthermore,

$$
C \cdot C=\int_{X} \eta_{C}^{\prime} \wedge \eta_{C}=\int_{\Sigma_{0}}\left(f_{\epsilon}^{\prime}\right)^{*} \eta_{C}+\sum_{i=1}^{k} \frac{1}{m_{i}} \int_{\widehat{D_{i}}}\left(f_{i, \epsilon}^{\prime}\right)^{*} \eta_{C} .
$$


By way of construction,

$$
\int_{\Sigma_{0}}\left(f_{\epsilon}^{\prime}\right)^{*} \eta_{C}=c_{1}\left(\nu_{\epsilon}, \tau_{v}\right)\left(\Sigma_{0}, \partial \Sigma_{0}\right)+\sum_{\left\{\left[z, z^{\prime}\right] \mid z \neq z^{\prime}, f_{\epsilon}(z)=f_{\epsilon}\left(z^{\prime}\right)\right\}} 2 t_{\left[z, z^{\prime}\right]},
$$

where $\left[z, z^{\prime}\right]$ denotes the unordered pair of $z, z^{\prime}$, and $t_{\left[z, z^{\prime}\right]}$ is the order of tangency of the intersection $f_{\epsilon}(z)=f_{\epsilon}\left(z^{\prime}\right)$. It is easily seen that the second term in the above equation is equal to

$$
\sum_{\left\{\left[z, z^{\prime}\right] \mid z \neq z^{\prime}, f(z)=f\left(z^{\prime}\right)\right\}} 2 k_{\left[z, z^{\prime}\right]}+\sum_{\{z \mid d f(z)=0\}} 2 k_{z}, \text { where } z, z^{\prime} \in \Sigma_{0} .
$$

To evaluate $\int_{\widehat{D_{i}}}\left(f_{i, \epsilon}^{\prime}\right)^{*} \eta_{C}, i=1,2, \cdots, k$, let $I_{i}$ be the set labeling $\Lambda(C)_{z_{i}}$, ie $\Lambda(C)_{z_{i}}=\left\{C_{i, \alpha} \mid \alpha \in I_{i}\right\}$, and let $C_{i} \in \Lambda(C)_{z_{i}}$ be the element defined by $f_{i}$. Then

$$
\begin{aligned}
& \int_{\widehat{D_{i}}}\left(f_{i, \epsilon}^{\prime}\right)^{*} \eta_{C}=\int_{\widehat{D_{i}}}\left(f_{i, \epsilon}^{\prime}\right)^{*}\left(\sum_{\left\{j \mid f\left(z_{i}\right)=f\left(z_{j}\right)\right\}} \frac{1}{m_{j}} \sum_{g \in G_{i}} g^{*} \overline{\Theta_{j, \epsilon}}\right) \\
& =\frac{1}{m_{i}} \sum_{g \in G_{i}} \int_{\widehat{D_{i}}}\left(f_{i, \epsilon}^{\prime}\right)^{*}\left(g^{*} \overline{\Theta_{i, \epsilon}}\right) \\
& +\sum_{\left\{j \neq i \mid f\left(z_{j}\right)=f\left(z_{i}\right)\right\}} \frac{1}{m_{j}} \sum_{g \in G_{i}} \int_{\widehat{D_{i}}}\left(f_{i, \epsilon}^{\prime}\right)^{*}\left(g^{*} \overline{\Theta_{j, \epsilon}}\right) \\
& =c_{1}\left(\nu_{i, \epsilon}, \tau_{i, v}\right)\left(\widehat{D_{i}}, \partial \widehat{D_{i}}\right)+C_{i} \cdot C_{i}+\sum_{\alpha \in I_{i}} C_{i} \cdot C_{i, \alpha} \\
& +\sum_{\left\{j \neq i \mid f\left(z_{i}\right)=f\left(z_{j}\right)\right\}} \sum_{\beta \in I_{j}} C_{i} \cdot C_{j, \beta} \\
& =c_{1}\left(\nu_{i, \epsilon}, \tau_{i, v}\right)\left(\widehat{D_{i}}, \partial \widehat{D_{i}}\right)+\frac{m_{i}}{\left|G_{i}\right|}\left(\sum_{\alpha \in I_{i}} C_{i, \alpha} \cdot C_{i, \alpha}+\sum_{\alpha, \beta \in I_{i}} C_{i, \alpha} \cdot C_{i, \beta}\right) \\
& +\frac{m_{i}}{\left|G_{i}\right|} \sum_{\left\{j \neq i \mid f\left(z_{i}\right)=f\left(z_{j}\right)\right\}} \sum_{\alpha \in I_{i}, \beta \in I_{j}} C_{i, \alpha} \cdot C_{j, \beta} .
\end{aligned}
$$

In order to evaluate $c_{1}\left(\nu_{i, \epsilon}, \tau_{i, v}\right)\left(\widehat{D_{i}}, \partial \widehat{D_{i}}\right)$, we observe that $f_{i, \epsilon}$ is an immersion and equals $\left(z^{l_{i}}, 0\right)+O\left(|z|^{l_{i}+1}\right)$ near $\partial \widehat{D_{i}}$. Let $\tau_{i, v}^{\prime}$ be the trivialization of $\nu_{i, \epsilon}$ along $\partial \widehat{D_{i}}$ which can be extended over the entire $\widehat{D_{i}}$. Then $\tau_{i, v}^{\prime}$ is given by the section $\left(0, z^{-l_{i}+1}\right)$ up to homotopy. But $\tau_{i, v}$ is given by the section $\left(0, z^{-l_{i}+m_{i, 1}+m_{i, 2}}\right)$ up to homotopy. Hence

$$
c_{1}\left(\nu_{i, \epsilon}, \tau_{i, v}\right)\left(\widehat{D_{i}}, \partial \widehat{D_{i}}\right)=m_{i, 1}+m_{i, 2}-1 .
$$


Putting things altogether, we have

$$
\begin{aligned}
C \cdot C+c(C)= & c(C)+c_{1}\left(\nu_{\epsilon}, \tau_{v}\right)\left(\Sigma_{0}, \partial \Sigma_{0}\right)+\sum_{i=1}^{k} \frac{m_{i, 1}+m_{i, 2}-1}{m_{i}} \\
& +\sum_{\left\{\left[z, z^{\prime}\right] \mid z \neq z^{\prime}, f(z)=f\left(z^{\prime}\right)\right\}} 2 k_{\left[z, z^{\prime}\right]}+\sum_{z \in \Sigma} 2 k_{z} \\
= & -c_{1}\left(T \Sigma_{0}, \tau_{h}\right)\left(\Sigma_{0}, \partial \Sigma_{0}\right)-\sum_{i=1}^{k} \frac{1}{m_{i}} \\
& +\sum_{\left\{\left[z, z^{\prime}\right] \mid z \neq z^{\prime}, f(z)=f\left(z^{\prime}\right)\right\}} 2 k_{\left[z, z^{\prime}\right]}+\sum_{z \in \Sigma} 2 k_{z} \\
= & 2 g_{|\Sigma|}-2+k-\sum_{i=1}^{k} \frac{1}{m_{i}} \\
& +\sum_{\left\{\left[z, z^{\prime}\right] \mid z \neq z^{\prime}, f(z)=f\left(z^{\prime}\right)\right\}} 2 k_{\left[z, z^{\prime}\right]}+\sum_{z \in \Sigma} 2 k_{z},
\end{aligned}
$$

from which the adjunction formula for the case where $C$ is of type I follows easily.

The case where $C$ is of type II is actually much simpler. It follows by directly evaluating the last integral in

$$
C \cdot C=\int_{X} \eta_{C} \wedge \eta_{C}=\int_{\Sigma} f^{*} \eta_{C}
$$

and then appealing to $c_{1}(T X)(\Sigma)=c_{1}(\nu)(\Sigma)+c_{1}(T \Sigma)(\Sigma)$ and $m_{C}=m_{\Sigma}$.

\section{Proof of Theorem 3.2}

For simplicity, we shall only consider the case where $C, C^{\prime}$ are of type I. The discussion for the rest of the cases is similar, and we shall leave the details to the reader.

Let $\eta_{C}, \eta_{C^{\prime}}$ be the closed 2 -forms in Lemma 3.5 which are Poicaré dual to the differentiable cycles $f(\Sigma), f^{\prime}\left(\Sigma^{\prime}\right)$ respectively. Then

$$
\begin{aligned}
C \cdot C^{\prime} & =\int_{X} \eta_{C} \wedge \eta_{C^{\prime}} \\
& =\int_{\Sigma_{0}} f_{\epsilon}^{*} \eta_{C^{\prime}}+\sum_{i=1}^{k} \frac{1}{m_{i}} \int_{\widehat{D_{i}}} f_{i, \epsilon}^{*} \eta_{C^{\prime}} .
\end{aligned}
$$


Now observe that the subset $\left\{\left(z, z^{\prime}\right) \mid f(z)=f^{\prime}\left(z^{\prime}\right)\right\} \subset \Sigma \times \Sigma^{\prime}$ is finite. Hence we may arrange in the construction of $\eta_{C}$ and $\eta_{C^{\prime}}$ such that for sufficiently small $\epsilon>0, \int_{\Sigma_{0}} f_{\epsilon}^{*} \eta_{C^{\prime}}$ equals $\sum k_{\left(z, z^{\prime}\right)}$ where $\left(z, z^{\prime}\right)$ is running over the set of pairs with $f(z)=f^{\prime}\left(z^{\prime}\right)$ being a regular point of $X$, and $\sum_{i=1}^{k} \frac{1}{m_{i}} \int_{\widehat{D_{i}}} f_{i, \epsilon}^{*} \eta_{C^{\prime}}$ equals $\sum k_{\left(z, z^{\prime}\right)}$ where $\left(z, z^{\prime}\right)$ is running over the set of pairs with $f(z)=f^{\prime}\left(z^{\prime}\right)$ being an orbifold point of $X$. Hence the theorem.

\section{Proof of main results}

We begin by setting the stage. Let $p, q$ be relatively prime integers with $0<$ $q<p$. We denote by $C_{(p, q)}$ the symplectic cone over $L(p, q)$, which is the symplectic orbifold $\left(\mathbb{C}^{2}, \omega_{0}\right) / \mathbb{Z}_{p}$ where $\omega_{0}=\frac{\sqrt{-1}}{2} \sum_{i=1}^{2} d z_{i} \wedge d \bar{z}_{i}$ and $\mathbb{Z}_{p}$ acts by $\mu_{p} \cdot\left(z_{1}, z_{2}\right)=\left(\mu_{p} z_{1}, \mu_{p}^{q} z_{2}\right)$. Let $d$ be the descendant of the function $\frac{1}{2}\left(\left|z_{1}\right|^{2}+\right.$ $\left.\left|z_{2}\right|^{2}\right)$ on $\mathbb{C}^{2}$ to $C_{(p, q)}$. Then for any $r>0, C_{(p, q)}(r) \equiv d^{-1}([0, r]) \subset C_{(p, q)}$ is a suborbifold of contact boundary $\left(L(p, q), \xi_{0}\right)$.

Next we follow the discussion in 12 to embed each $C_{(p, q)}(r)$ into an appropriate closed symplectic 4 -orbifold. To this end, consider the Hamiltonian circle action on $\left(\mathbb{C}^{2}, \omega_{0}\right)$

$$
s \cdot\left(z_{1}, z_{2}\right)=\left(s z_{1}, s^{p+q} z_{2}\right), \forall s \in \mathbb{S}^{1} \equiv\{z \in \mathbb{C}|| z \mid=1\},
$$

with the Hamiltonian function given by $\mu\left(z_{1}, z_{2}\right)=\frac{1}{2}\left(\left|z_{1}\right|^{2}+(p+q)\left|z_{2}\right|^{2}\right)$. It is easily seen that the $\mathbb{Z}_{p}$-action on $\mathbb{C}^{2}$ is the action induced from the circle action by $\mathbb{Z}_{p} \subset \mathbb{S}^{1}$, thus there is a corresponding Hamiltonian circle action on $\mathbb{C}^{2} / \mathbb{Z}_{p}=C_{(p, q)}$ with the Hamiltonian function given by $\mu^{\prime} \equiv \frac{1}{p} \mu$. According to [12], for any $R>0$, there is a symplectic 4-orbifold, denoted by $X_{(p, q)}(R)$, which is obtained from $\left(\mu^{\prime}\right)^{-1}([0, R])$ by collapsing each orbit of the circle action on $\left(\mu^{\prime}\right)^{-1}(R)$ to a point. It is clear that for any $R>\frac{1}{p}(p+q) r, C_{(p, q)}(r)$ is a suborbifold of $X_{(p, q)}(R)$ of contact boundary $\left(L(p, q), \xi_{0}\right)$. Furthermore, there is a distinguished 2-dimensional symplectic suborbifold $C_{0} \equiv\left(\mu^{\prime}\right)^{-1}(R) / \mathbb{S}^{1} \subset$ $X_{(p, q)}(R)$, whose normal bundle has Euler number $\frac{p}{p+q}$, and whose orbifold genus is $\frac{1}{2}-\frac{1}{2(p+q)}$, cf Section 3 .

Now let $(W, \omega)$ be a symplectic cobordism from $\left(L\left(p^{\prime}, q^{\prime}\right), \xi_{0}^{\prime}\right)$ to $\left(L(p, q), \xi_{0}\right)$. By adding appropriate "symplectic collars" to the two ends of $W$, which does not change the diffeomorphism class of $W$, we may assume without loss of generality that a neighborhood of $L\left(p^{\prime}, q^{\prime}\right)$ in $W$ is identified with a neighborhood of $\partial C_{\left(p^{\prime}, q^{\prime}\right)}\left(r^{\prime}\right)$ in $C_{\left(p^{\prime}, q^{\prime}\right)} \backslash \operatorname{int}\left(C_{\left(p^{\prime}, q^{\prime}\right)}\left(r^{\prime}\right)\right)$ for some $r^{\prime}>0$, and a neighborhood of $L(p, q)$ in $W$ is identified with a neighborhood of $\partial C_{(p, q)}(r)$ in $C_{(p, q)}(r)$ for some 
$r>0$. Consequently, we can close up $W$ by gluing $X_{(p, q)}(R) \backslash C_{(p, q)}(r)$ and $C_{\left(p^{\prime}, q^{\prime}\right)}\left(r^{\prime}\right)$ onto the corresponding ends of $W$ for some fixed $R>\frac{1}{p}(p+q) r$. We denote by $(X, \omega)$ the resulting symplectic 4 -orbifold. Note that there is a distinguished 2-dimensional symplectic suborbifold $C_{0} \subset X$ inherited from $C_{0} \subset X_{(p, q)}(R)$.

With the preceding understood, the strategy for proving Theorem 1.1 is to construct a diffeomorphism of orbifold pairs from $\left(X, C_{0}\right)$ to $\left(X_{(p, q)}(R), C_{0}\right)$.

First of all, some preliminary information about $\left(X, C_{0}, \omega\right)$. The orbifold $X$ has two singular points, one of them, denoted by $x^{\prime}$, is inherited from $C_{\left(p^{\prime}, q^{\prime}\right)}\left(r^{\prime}\right)$ and has type $\left(p^{\prime}, q^{\prime}\right)$, and the other, denoted by $x$, is inherited from $X_{(p, q)}(R) \backslash$ $C_{(p, q)}(r)$ and has type $(p+q, p)$. Here a singular point has type $(a, b)$ if the isotropy group is $\mathbb{Z}_{a}$ with action on a local uniformizing system given by $\mu_{a}$. $\left(z_{1}, z_{2}\right)=\left(\mu_{a} z_{1}, \mu_{a}^{b} z_{2}\right)$. The suborbifold $C_{0}$ has only one orbifold point, the point $x$ with order $p+q$, and is given locally by $z_{2}=0$ on the local uniformizing system. We fix an $\omega$-compatible almost complex structure $J$ on $X$, such that the suborbifold $C_{0}$ is $J$-holomorphic. For convenience, we assume that $J$ is integrable near $x, x^{\prime}$. (This is possible because of the equivariant Darboux' theorem.) By the discussion in Section 3, we see that $C_{0} \cdot C_{0}=e(\nu)\left(C_{0}\right)=\frac{p}{p+q}$, where $e(\nu)$ is the Euler class of the normal bundle $\nu$ of $C_{0}$ in $X$. On the other hand, by the adjunction formula in Theorem 3.1, we have

$$
c_{1}\left(K_{X}\right)\left(C_{0}\right)=2\left(\frac{1}{2}-\frac{1}{2(p+q)}\right)-2-C_{0} \cdot C_{0}=-\frac{2 p+q+1}{p+q}
$$

for the canonical bundle $K_{X}$ of the almost complex 4 -orbifold $(X, J)$.

Next we digress on the Fredholm theory for pseudoholomorphic curves in a symplectic 4 -orbifold $(X, \omega)$. To this end, for any given orbifold Riemann surface $\Sigma$, we fix a sufficiently large positive integer $k$, and consider $[\Sigma ; X]$, the space of $C^{k}$ maps from $\Sigma$ into $X$. It is shown in 3. (Part I, Theorem $1.4)$ that $[\Sigma ; X]$ is a smooth Banach orbifold (Hausdorff and second countable). Moreover, a map $f \in[\Sigma ; X]$ is a smooth point in the Banach orbifold if $\operatorname{Im} f$ contains a regular point of $X$. Thus for the purpose here we may assume for simplicity that $\Sigma$ is reduced and $[\Sigma ; X]$ is a Banach manifold. The tangent space $T_{f}$ at $f \in[\Sigma ; X]$ is the space of $C^{k}$ sections of $f^{*}(T X)$, the pullback bundle of $T X$ via $f$.

For any $f \in[\Sigma ; X]$, let $\mathcal{E}_{f}$ be the subspace of the space of $C^{k-1}$ sections of the orbifold vector bundle $\operatorname{Hom}\left(T \Sigma, f^{*}(T X)\right) \rightarrow \Sigma$, which consists of sections $s$ satisfying $s \circ j=-J \circ s$ for a fixed choice of $\omega$-compatible almost complex structure $J$ on $X$ and the complex structure $j$ on $\Sigma$. Then there is a Banach 
bundle $\mathcal{E}$ over $[\Sigma ; X]$ whose fiber at $f$ is $\mathcal{E}_{f}$. Consider the smooth section $\underline{L}:[\Sigma ; X] \rightarrow \mathcal{E}$ defined by

$$
\underline{L}(f) \equiv d f+J \circ d f \circ j .
$$

The zero loci $\underline{L}^{-1}(0)$ is the space of $J$-holomorphic maps from $\Sigma$ into $X$. By elliptic regularity, each map in $\underline{L}^{-1}(0)$ is a $C^{\infty}$ map. Moreover, $\underline{L}$ is a Fredholm section, and its linearization $D \underline{L}$ at each $f \in \underline{L}^{-1}(0)$ is given by a formula

$$
D \underline{L}_{f}(u)=L_{f}(u), \quad u \in T_{f},
$$

where $L_{f}: T_{f} \rightarrow \mathcal{E}_{f}$ is an elliptic linear differential operator of Cauchy-Riemann type, whose coefficients are smooth functions on $\Sigma$ which depend on $f$ smoothly. The following facts are crucial for the consideration of surjectivity of $D \underline{L}$.

- When $J$ is integrable in a neighborhood of $\operatorname{Im} f$ and $f$ is $J$-holomorphic, $D \underline{L}_{f}=L_{f}$ is the usual $\bar{\partial}$-operator for the orbifold holomorphic vector bundle $f^{*}(T X)$ over $\Sigma$.

- When $f$ is a multiplicity-one parametrization of a $J$-holomorphic suborbifold $C$, the linearization $D \underline{L}_{f}=L_{f}$ is surjective when $c_{1}(T C)(C)>0$ and $c_{1}\left(K_{X}\right)(C)<0$. This is the orbifold analog of the regularity criterion discussed in Lemma 3.3.3 of [16].

The index of $D \underline{L}_{f}=L_{f}$ can be computed using the index formula of Kawasaki [10] for elliptic operators on orbifolds, cf Lemma 3.2.4 in [4].

To state the formula, let $z_{1}, z_{2}, \cdots, z_{l}$ be the set of orbifold points of $\Sigma$ with orders $m_{1}, m_{2}, \cdots, m_{l}$ respectively. Moreover, suppose at each $z_{i}$, a local representative of $f$ is given by $\left(f_{i}, \rho_{i}\right):\left(\widehat{D_{i}}, \mathbb{Z}_{m_{i}}\right) \rightarrow\left(\widehat{V}_{i}, G_{i}\right)$ where $\rho_{i}\left(\mu_{m_{i}}\right)$ acts on $\widehat{V}_{i}$ by $\rho_{i}\left(\mu_{m_{i}}\right) \cdot\left(w_{1}, w_{2}\right)=\left(\mu_{m_{i}}^{m_{i, 1}} w_{1}, \mu_{m_{i}}^{m_{i, 2}} w_{2}\right), 0 \leq m_{i, 1}, m_{i, 2}<m_{i}$. With this understood, Index $D \underline{L}_{f}=2 d$ where $d \in \mathbb{Z}$ is given by

$$
d=c_{1}(T X) \cdot[f(\Sigma)]+2-2 g_{|\Sigma|}-\sum_{i=1}^{l} \frac{m_{i, 1}+m_{i, 2}}{m_{i}} .
$$

(Here $g_{|\Sigma|}$ is the genus of the underlying Riemann surface.) End of digression.

Now let $\Sigma$ be the orbifold Riemann sphere with one orbifold point $z_{\infty} \equiv \infty$ of order $p+q$. Observe that as a complex analytic space, $\Sigma$ is biholomorphic to the underlying Riemann sphere $|\Sigma|$, hence it has a unique complex structure. Moreover, the group of automorphisms $G$ can be naturally identified with the subgroup of the automorphism group of $|\Sigma|$ which fixes the point $\infty$. Note that $|\Sigma| \backslash\{\infty\}=\mathbb{C}$, so that $G$ can be identified with the group $\left\{(a, b) \in C^{*} \times \mathbb{C} \mid\right.$ $z \mapsto a z+b\}$ of linear translations on $\mathbb{C}$. 
We shall consider the moduli space $\widetilde{\mathcal{M}}$ of $J$-holomorphic maps $f: \Sigma \rightarrow X$ which obey

- $[f(\Sigma)]=\left[C_{0}\right]$ in $H_{2}(X ; \mathbb{Q})$,

- $f\left(z_{\infty}\right)=x$, and in a local representative $\left(f_{\infty}, \rho_{\infty}\right)$ of $f$ at $z_{\infty}, \rho_{\infty}\left(\mu_{(p+q)}\right)$ $=\mu_{(p+q)}$, which acts by $\left(z_{1}, z_{2}\right) \mapsto\left(\mu_{(p+q)} z_{1}, \mu_{(p+q)}^{p} z_{2}\right)$. (Here $z_{1}, z_{2}$ are holomorphic coordinates on a local uniformizing system at $x$ in which $C_{0}$ is locally given by $z_{2}=0$.)

We set $\mathcal{M}=\widetilde{\mathcal{M}} / G$ for the corresponding moduli space of unparametrized $J$-holomorphic maps, where $G$ acts on $\widetilde{\mathcal{M}}$ by reparametrization.

With the preceding understood, consider the following:

Lemma 4.1 Suppose $W$ is a (symplectic) homology cobordism. (Note that in particular, $p=p^{\prime}$ and $H_{2}(X ; \mathbb{Q})=\mathbb{Q} \cdot\left[C_{0}\right]$.) Then

(1) Each member of $\widetilde{\mathcal{M}}$ is either an orbifold embedding onto a suborbifold in $X$, or is a multiply covered map with multiplicity $p$ onto a suborbifold containing both $x, x^{\prime}$. Moreover, in the latter case, either $q^{\prime}=q$ or $q^{\prime} q \equiv 1$ $(\bmod p)$ must be satisfied, and there is at most one such a member of $\widetilde{\mathcal{M}}$ up to reparametrization by elements of $G$.

(2) One may alter $J$ appropriately such that $C_{0}$ is still $J$-holomorphic, and $\widetilde{\mathcal{M}}$ is a smooth manifold of dimension 6 . Furthermore, $\mathcal{M}$ is a compact, closed, 2 dimensional smooth orbifold (possibly disconnected) with at most one orbifold point of order $p$, and the action of $G$ on $\widetilde{\mathcal{M}}$ defines a smooth orbifold principal $G$-bundle $\widetilde{\mathcal{M}} \rightarrow \mathcal{M}$.

Before proving Lemma 4.1, let us observe the following:

Lemma 4.2 Let $C$ be any $J$-holomorphic curve in $X$ such that

- $C$ contains both singular points,

- $[C]=r\left[C_{0}\right]$ for some $r \in(0,1] \cap \mathbb{Q}$.

Then $C$ is a suborbifold and $[C]=\frac{1}{p}\left[C_{0}\right]$. Moreover, there is at most one such $J$-holomorphic curves in $X$.

Proof First of all, we claim $r \geq \frac{1}{p}$. To see this, note that $C \neq C_{0}$ because $C$ contains both singular points. By the intersection formula (cf Theorem 3.2),

$$
r \cdot \frac{p}{p+q}=C \cdot C_{0} \geq \frac{1}{p+q}
$$


which verifies the claim.

Now let $f: \Sigma \rightarrow X$ be a multiplicity-one parametrization of $C$, and $z_{0}, z_{0}^{\prime} \in \Sigma$ be any points such that $f\left(z_{0}\right)=x, f\left(z_{0}^{\prime}\right)=x^{\prime}$. Let $m_{0}, m_{0}^{\prime}$ be the order of $z_{0}, z_{0}^{\prime}$ respectively. Then observe that if $m_{0}<p+q$ (resp. $m_{0}^{\prime}<p$ ), the contribution $k_{z_{0}}\left(\right.$ resp. $\left.k_{z_{0}^{\prime}}\right)$ on the right hand side of the adjunction formula for $C$ (cf Theorem 3.1) is no less than $\frac{1}{2 m_{0}}$ (resp. $\frac{1}{2 m_{0}^{\prime}}$ ). (Here is the calculation for the case of $m_{0}: k_{z_{0}} \geq \frac{1}{2(p+q)} \cdot\left[\frac{p+q}{m_{0}}\left(\frac{p+q}{m_{0}}-1\right)\right] \geq \frac{1}{2 m_{0}}$ if $m_{0}<p+q$.) It follows easily that the right hand side of the adjunction formula for $C$ is no less than

$$
\frac{1}{2}\left(1-\frac{1}{p+q}\right)+\frac{1}{2}\left(1-\frac{1}{p}\right)
$$

which has an equality only if $m_{0}=p+q$ and $m_{0}^{\prime}=p$.

On the other hand, the left hand side of the adjunction formula for $C$, the virtual genus $g(C)$, equals

$$
\frac{1}{2}\left(\frac{p}{p+q} \cdot r^{2}-\frac{2 p+q+1}{p+q} \cdot r\right)+1 .
$$

As a function of $r$, it is decreasing over $(0,1]$, hence the maximum of $g(C)$ is attained at $r=\frac{1}{p}$, and it equals

$$
\frac{1}{2}\left(\frac{p}{p+q} \cdot\left(\frac{1}{p}\right)^{2}-\frac{2 p+q+1}{p+q} \cdot \frac{1}{p}\right)+1=\frac{1}{2}\left(1-\frac{1}{p+q}\right)+\frac{1}{2}\left(1-\frac{1}{p}\right) .
$$

By the adjunction formula, $C$ is a suborbifold and $[C]=\frac{1}{p}\left[C_{0}\right]$.

To see that there is at most one such $J$-holomorphic curves, note that if there were two distinct such curves, the algebraic intersection number, which is $\frac{p}{p^{2}(p+q)}$, would be at least $\frac{1}{p+q}+\frac{1}{p}$ by the intersection formula. A contradiction.

\section{Proof of Lemma 4.1}

(1) By the adjunction formula, each multiplicity-one member $f \in \widetilde{\mathcal{M}}$ must be an orbifold embedding onto a suborbifold. Now suppose $f \in \widetilde{\mathcal{M}}$ is multiply covered with multiplicity $m>1$. Let $C$ be the corresponding $J$-holomorphic curve. Then $[C]=\frac{1}{m}\left[C_{0}\right]<\left[C_{0}\right]$, which implies that $C$ also contains the other singular point $x^{\prime}$. This is because by the assumption, $W$ is a homology cobordism, so that $H_{2}\left(X \backslash\left\{x^{\prime}\right\} ; \mathbb{Z}\right)$ is generated by the class of $C_{0}$, and hence $C$ can not be contained entirely in $X \backslash\left\{x^{\prime}\right\}$. By Lemma 4.2, $f$ has multiplicity $p$, and $C$ is a suborbifold, which is unique in such kind. 
To complete the proof of (1), it remains to show that either $q^{\prime}=q$ or $q^{\prime} q \equiv 1$ $(\bmod p)$ if there is indeed such a curve $C$.

To this end, let $f: \Sigma \rightarrow X$ be any multiplicity-one parametrization of $C$, and $z_{0}, z_{0}^{\prime} \in \Sigma$ be the points such that $f\left(z_{0}\right)=x, f\left(z_{0}^{\prime}\right)=x^{\prime}$. Since $C \neq C_{0}$ and $C \cdot C_{0}=\frac{1}{p} \cdot \frac{p}{p+q}=\frac{1}{p+q}$, it follows easily that the local representative of $f$ at $z_{0}$ must be in the form $\left((u(z), z), \rho_{0}\right)$ for some holomorphic function $u$ and the isomorphism $\rho_{0}$ where $\rho_{0}\left(\mu_{(p+q)}\right)=\mu_{(p+q)}^{l}$ with $p l \equiv 1(\bmod p+q)$. On the other hand, the local representative of $f$ at $z_{0}^{\prime}$ could either be $\left((w(z), z), \rho_{0}^{\prime}\right)$, where $\rho_{0}^{\prime}\left(\mu_{p}\right)=\mu_{p}^{l^{\prime}}$ with $l^{\prime} q^{\prime} \equiv 1(\bmod p)$, or $\left((z, w(z)), \rho_{0}^{\prime}\right)$ with $\rho_{0}^{\prime}\left(\mu_{p}\right)=\mu_{p}$. Assuming the former case, we have, by the index formula for $D \underline{L}_{f}$,

$$
\frac{2 p+q+1}{p(p+q)}+2-\frac{l+1}{p+q}-\frac{l^{\prime}+1}{p} \in \mathbb{Z},
$$

which implies that $r(p+q)-q l^{\prime} \equiv 0(\bmod p)$ with $r$ given by the equation $1-l p=r(p+q)$. It is easily seen that in this case, $q l^{\prime} \equiv q r \equiv 1(\bmod p)$, and hence $q^{\prime}=q$ because $l^{\prime} q^{\prime} \equiv 1(\bmod p)$. Similarly, the latter case implies $q^{\prime} q \equiv 1(\bmod p)$.

(2) For the smoothness of $\widetilde{\mathcal{M}}$, we need to show that for any $f \in \widetilde{\mathcal{M}}$, the linearization $D \underline{L}_{f}$ is surjective. The dimension of $\widetilde{\mathcal{M}}$ is the index of $D \underline{L}_{f}$, $f \in \widetilde{\mathcal{M}}$, which is easily seen to be 6 by the index formula for $D \underline{L}_{f}$.

By the regularity criterion we mentioned earlier, $\widetilde{\mathcal{M}}$ is smooth at each $f$ which is not multiply covered, because for any such an $f, C \equiv \operatorname{Im} f$ is a suborbifold satisfing $c_{1}(T C)(C)=2-\left(1-\frac{1}{p+q}\right)>0$ and $c_{1}\left(K_{X}\right)(C)=-\frac{2 p+q+1}{p+q}<0$. Suppose there is a multiply covered member (which is the only one up to reparametrization by (1)), and let $C_{0}^{\prime}$ be the corresponding $J$-holomorphic curve. We consider the weighted projective space $\mathbb{P}(1, p, p+q)$, which is the quotient of $\mathbb{S}^{5}$ under the $\mathbb{S}^{1}$-action

$$
s \cdot\left(z_{1}, z_{2}, z_{3}\right)=\left(s z_{1}, s^{p} z_{2}, s^{p+q} z_{3}\right), \forall s \in \mathbb{S}^{1} .
$$

It is easily seen that a regular neighborhood of $C_{0}^{\prime}$ in $X$ is diffeomorphic to a regular neighborhood of $\mathbb{P}(p, p+q)$ in $\mathbb{P}(1, p, p+q)$, where $\mathbb{P}(p, p+q)$ is defined by $z_{1}=0$. According to [2, $\mathbb{P}(1, p, p+q)$ has an orbifold Kähler metric of positive Ricci curvature. By the orbifold version of symplectic neighborhood theorem, we can alter the almost complex structure $J$ in a regular neighborhood of $C_{0}^{\prime}$ such that $\omega(\cdot, J(\cdot))$ is Kähler of positive Ricci curvature. (Note that we can arrange so that $C_{0}$ is still $J$-holomorphic, and $J$ is integrable near singular points $x, x^{\prime}$.) With this understood, for any $f \in \widetilde{\mathcal{M}}$ parametrizing $C_{0}^{\prime}, D \underline{L}_{f}$ is the usual $\bar{\partial}$-operator for the orbifold holomorphic vector bundle $f^{*}(T X)$ over 
$\Sigma$. In this case, the surjectivity of $D \underline{L}_{f}$ follows from the orbifold version of a Bochner type vanishing theorem for negative holomorphic vector bundles (cf [11]). Thus in any event, by altering $J$ if necessary, we can arrange so that $\widetilde{\mathcal{M}}$ is a smooth manifold.

The action of $G$ on $\widetilde{\mathcal{M}}$ is smooth (see the general discussion at the end of $\S 3.3$ of Part I of [3]), and is free at each $f \in \widetilde{\mathcal{M}}$ which is not multiply covered. At a multiply covered $f \in \widetilde{\mathcal{M}}$, the isotropy subgroup is the cyclic subgroup $\left\{\left(\mu_{p}^{l}, 0\right) \mid l=0, \cdots, p-1\right\} \subset G$ of order $p$ up to conjugation. (Note that $p$ equals the multiplicity of the covering.) Thus $\widetilde{\mathcal{M}} \rightarrow \widetilde{\mathcal{M}} / G=\mathcal{M}$ is a smooth orbifold principal $G$-bundle over a smooth 2-dimensional orbifold with at most one orbifold point of order $p$.

It remains to show that $\mathcal{M}$ is compact. First of all, by the orbifold version of the Gromov's compactness theorem (cf [7, 18, 21]) which was proved in [4, any sequence of maps $f_{n} \in \widetilde{\mathcal{M}}$ has a subsequence which converges to a cuspcurve after suitable reparametrization. More concretely, after reparametrization if necessary, there is a subsequence of $f_{n}$, which is still denoted by $f_{n}$ for simplicity, and there are at most finitely many simple closed loops $\gamma_{1}, \cdots, \gamma_{l} \subset$ $\Sigma$ containing no orbifold points, and a nodal orbifold Riemann surface $\Sigma^{\prime}=$ $\cup_{\omega} \Sigma_{\omega}$ obtained by collapsing $\gamma_{1}, \cdots, \gamma_{l}$, and a $J$-holomorphic map $f: \Sigma^{\prime} \rightarrow X$, such that (1) $f_{n}$ converges in $C^{\infty}$ to $f$ on any given compact subset in the complement of $\gamma_{1}, \cdots, \gamma_{l},(2)\left[f_{n}(\Sigma)\right]=\left[f\left(\Sigma^{\prime}\right)\right] \in H_{2}(X ; \mathbb{Q})$, and $(3) f \in \widetilde{\mathcal{M}}$ and $f_{n}$ converges to $f$ in $C^{\infty}$ if there is only one component of $\Sigma^{\prime}=\cup_{\omega} \Sigma_{\omega}$ over which $f$ is nonconstant.

Hence the space $\mathcal{M}$ is compact if there is only one component of $\Sigma^{\prime}=\cup_{\omega} \Sigma_{\omega}$ over which $f$ is nonconstant. Suppose this is not true. Then there is a nonconstant component $\left.f_{\omega} \equiv f\right|_{\Sigma_{\omega}}: \Sigma_{\omega} \rightarrow X$, where $\Sigma_{\omega}$ is obtained by collapsing a simple closed loop $\gamma \in\left\{\gamma_{1}, \cdots, \gamma_{l}\right\}$ which bounds a disc $D \subset \Sigma$, such that $z_{\infty} \in \Sigma \backslash D$ and $f_{n}$ converges to $f_{\omega}$ in $C^{\infty}$ on any compact subset of the interior of $D$. Set $C_{\omega} \equiv \operatorname{Im} f_{\omega}$. Since we assume that there are more than one nonconstant components, $\left[C_{\omega}\right] \leq\left[f_{\omega}\left(\Sigma_{\omega}\right)\right]<\left[C_{0}\right]$ must hold. (Note that $\left.H_{2}(X ; \mathbb{Q})=\mathbb{Q} \cdot\left[C_{0}\right].\right)$ By the assumption that $W$ is a homology cobordism, $C_{\omega}$ must contain the singular point $x^{\prime}$ as we argued earlier. We claim that $C_{\omega}$ must also contain the other singular point. Suppose not, then $C_{\omega} \neq C_{0}$, and $C_{\omega}$ must intersect with $C_{0}$ at a smooth point, because $C_{\omega} \cdot C \neq 0$. Then by the intersection formula, $C_{\omega} \cdot C_{0} \geq 1$, which implies that $\left[C_{\omega}\right]=r\left[C_{0}\right]$ for some $r \geq 1+\frac{q}{p}$. A contradiction to $\left[C_{\omega}\right]<\left[C_{0}\right]$. Now by Lemma 4.2, $C_{\omega}$ is a suborbifold and $\left[C_{\omega}\right]=\frac{1}{p}\left[C_{0}\right]$. 
On the other hand, observe that there is a regular point $z_{0} \in \Sigma_{\omega}$ such that either $f_{\omega}\left(z_{0}\right)=x$ or $f_{\omega}\left(z_{0}\right)=x^{\prime}$. Let $m_{\omega} \geq 1$ be the multiplicity of $f_{\omega}$, and let $D_{0}$ be a sufficiently small disc neighborhood of $z_{0}$ in $\Sigma_{\omega}$. Then it is easily seen that $m_{\omega}$ is no less than the degree of the covering map $\left.f_{\omega}\right|_{\partial D_{0}}$ onto the link of $f_{\omega}\left(z_{0}\right)$ in $C_{\omega}$, which is no less than $p+q$ or $p$, depending on whether $f_{\omega}\left(z_{0}\right)=x$ or $f_{\omega}\left(z_{0}\right)=x^{\prime}$. In any event, $m_{\omega} \geq p$. But this contradicts $\left[C_{\omega}\right]=\frac{1}{p}\left[C_{0}\right]$ as $\left[C_{\omega}\right]=\frac{1}{m_{\omega}}\left[f_{\omega}\left(\Sigma_{\omega}\right)\right]<\frac{1}{p}\left[C_{0}\right]$, because $\left[f_{\omega}\left(\Sigma_{\omega}\right)\right]<\left[C_{0}\right]$.

Hence there is only one nonconstant component, and therefore $\mathcal{M}$ is compact.

Let $H=\mathbb{C}^{*}$ be the subgroup of $G=\left\{(a, b) \in \mathbb{C}^{*} \times \mathbb{C}\right\}$ which consists of $\left\{(a, 0) \mid a \in \mathbb{C}^{*}\right\}$. We shall next find an appropriate reduction of $\widetilde{\mathcal{M}} \rightarrow \mathcal{M}$ to an orbifold principal $H$-bundle. We begin by giving a more detailed description of the orbifold structure on $\mathcal{M}$ and the orbifold principal $G$-bundle $\widetilde{\mathcal{M}} \rightarrow \mathcal{M}$.

First of all, we adopt the convention that $G$, as the automorphism group of $\Sigma$, acts on $\Sigma$ from the left. Second, for the orbifold structure on $\mathcal{M}$, we let $G$ act on $\widetilde{\mathcal{M}}$ from the left by defining $s \cdot f \equiv f \circ s^{-1}, \forall s \in G, f \in \widetilde{\mathcal{M}}$. (This is because the convention is that the group actions on a local uniformizing system are always from the left.) To describe the orbifold structure, recall that for any $f \in \widetilde{\mathcal{M}}$, there is a slice $S_{f}$ through $f$ which has the following properties (cf [1]):

- $S_{f} \subset \widetilde{\mathcal{M}}$ is a 2-dimensional disc containing $f$, which is invariant under the isotropy subgroup $G_{f}$ at $f$.

- For any $s \in G, s \cdot S_{f} \cap S_{f} \neq \emptyset$ iff $s \in G_{f}$.

- There exists an open neighborhood $\mathcal{O}$ of $1 \in G$ such that the map $\phi_{f}: \mathcal{O} \times S_{f} \rightarrow \widetilde{\mathcal{M}}$, defined by $(s, h) \mapsto s \cdot h$, is an open embedding.

Let $U \equiv \bigsqcup_{f \in \widetilde{\mathcal{M}}} S_{f}$ be the disjoint union of all slices. For any $h, h^{\prime} \in U$ which have the same orbit in $\mathcal{M}$, and for any $s \in G$ such that $s \cdot h=h^{\prime}$, let $\psi_{h^{\prime}, h}^{s}$ be the local self-diffeomorphism on $U$ defined as follows. Suppose $h \in S_{f}, h^{\prime} \in S_{f^{\prime}}$. Then there is an open neighborhood $O_{h} \subset S_{f}$ of $h$, invariant under the isotropy subgroup $G_{h}$ at $h$, such that $s \cdot O_{h} \subset \phi_{f^{\prime}}\left(\mathcal{O} \times S_{f^{\prime}}\right)$. Note that for any $g \in O_{h}$, there is a unique $s^{\prime} \in \mathcal{O}$ and a unique $g^{\prime} \in S_{f^{\prime}}$ such that $s \cdot g=\phi_{f^{\prime}}\left(s^{\prime}, g^{\prime}\right)=s^{\prime} \cdot g^{\prime}$. We define $\psi_{h^{\prime}, h}^{s}(g)=g^{\prime}$, which is clearly a local self-diffeomorphism on $U$ sending $h$ to $h^{\prime}$. The orbifold structure on $\mathcal{M}$ is given by the pseudogroup acting on $U$, which is generated by $\left\{\psi_{h^{\prime}, h}^{s}\right\}$.

To obtain the orbifold principal $G$-bundle $\widetilde{\mathcal{M}} \rightarrow \mathcal{M}$, we let $G$ act on $\widetilde{\mathcal{M}}$ from the right by defining $f \cdot s \equiv f \circ s, \forall s \in G, f \in \widetilde{\mathcal{M}}$. A local trivialization of 
$\widetilde{\mathcal{M}} \rightarrow \mathcal{M}$ over a slice $S_{f}$ is given by $\left(S_{f} \times G, G_{f}, \pi_{f}\right)$, where $G_{f}$ acts on $S_{f} \times G$ by $t \cdot(h, s)=(t \cdot h, t s), \forall t \in G_{f}$, and where $\pi_{f}: S_{f} \times G \rightarrow \widetilde{\mathcal{M}}$ sends $(h, s)$ to $h \cdot s=h \circ s$, which is invariant under the $G_{f}$-action (note that $t \cdot h=h \circ t^{-1}$ ). The transition function associated to each $\psi_{h^{\prime}, h}^{s}$ is given by $g \mapsto \bar{\psi}_{h^{\prime}, h}^{s}(g)$, $\forall g \in$ Domain $\left(\psi_{h^{\prime}, h}^{s}\right)$, with $\bar{\psi}_{h^{\prime}, h}^{s}(g): G \rightarrow G$ being the multiplication by $\left(s^{\prime}\right)^{-1} s$ from left, where $s^{\prime} \in \mathcal{O}$ is uniquely determined by $g \circ s^{-1}=\psi_{h^{\prime}, h}^{s}(g) \circ\left(s^{\prime}\right)^{-1}$.

In the same vein, by letting $H$ act on $\widetilde{\mathcal{M}}$ from the right, $\widetilde{\mathcal{M}}$ becomes an orbifold principal $H$-bundle over $\widetilde{\mathcal{M}} \times_{G}(G / H)$. A reduction of $\widetilde{\mathcal{M}} \rightarrow \mathcal{M}$ to an orbifold principal $H$-bundle is obtained by taking a smooth section of $\widetilde{\mathcal{M}} \times_{G}(G / H) \rightarrow \mathcal{M}$. Note that $G / H$ is naturally identified with $\mathbb{C}$, under which the coset $(a, b) H$ goes to $b \in \mathbb{C}$. Now at any possible multiply covered $f \in \widetilde{\mathcal{M}}, G_{f} \subset H$ iff $G_{f}$ is the cyclic subgroup generated by $\mu_{p}$. Its action on $G / H$ is given by $\mu_{p} \cdot(a, b) H=\left(\mu_{p}, 0\right)(a, b) H$, which is simply the multiplication by $\mu_{p}$ after identifying $G / H$ to $\mathbb{C}$. Hence for any such $f$, a local uniformizing system of $\widetilde{\mathcal{M}} \times{ }_{G}(G / H)$ at $(f, 0)$ is given by $\left(S_{f} \times \mathbb{C}, G_{f}\right)$, where $G_{f}$ acts by $\mu_{p} \cdot(h, b)=\left(\mu_{p} \cdot h, \mu_{p} b\right)$. To obtain a smooth section $u: \mathcal{M} \rightarrow \widetilde{\mathcal{M}} \times_{G}(G / H)$, we first pick a $G_{f}$-equivariant smooth section $u_{f}: S_{f} \rightarrow S_{f} \times \mathbb{C}$ for some arbitrary choice of a multiply covered $f$ with $G_{f} \subset H$ (note that if there is such an $f$, its orbit in $\mathcal{M}$ is unique, cf Lemma 4.1 (1)), then extend it to the rest of $\mathcal{M}$, where $\widetilde{\mathcal{M}} \times_{G}(G / H) \rightarrow \mathcal{M}$ is an ordinary fiber bundle with a contractible fiber $\mathbb{C}$. We denote by $\widehat{\mathcal{M}} \rightarrow \mathcal{M}$ the corresponding reduction to orbifold principal $H$-bundle. Note that $\widehat{\mathcal{M}}$ is naturally a 4 -dimensional submanifold of $\widetilde{\mathcal{M}}$.

Fixing a choice of the reduction $\widehat{\mathcal{M}} \rightarrow \mathcal{M}$, we let $Z \equiv \widehat{\mathcal{M}} \times{ }_{H} \mathbb{C}$ be the associated orbifold complex line bundle. Here $\mathbb{C}$ is canonically identified with $\Sigma \backslash\left\{z_{\infty}\right\}$, and hence the action of $H$ on $\mathbb{C}$ is given by complex multiplication.

There is a canonically defined smooth map of orbifolds $\psi: \widehat{\mathcal{M}} \times \Sigma \rightarrow X$, which induces the evaluation map $(f, z) \mapsto f(z)$ between the underlying spaces, cf Proposition 3.3.5 in Part I of [3]. Note that each trivialization $S_{f} \times \mathbb{C}$ of $Z \rightarrow \mathcal{M}$ over a slice $S_{f}$ is a submanifold of $\widehat{\mathcal{M}} \times \Sigma$, so that by restricting $\psi$ to $Z$, we obtain a smooth map of orbifolds Ev: $Z \rightarrow X$, which induces the evaluation map $[(f, z)] \mapsto f(z)$ between the underlying spaces.

Lemma 4.3 The map $E_{V}: Z \rightarrow X$ is a diffeomorphism of orbifolds onto $X \backslash\{x\}$.

Proof First of all, the map Ev induces an injective map on the underlying space. This is because each $J$-holomorphic curve parametrized by an $f \in$ 
$\widehat{\mathcal{M}}$ is a suborbifold, and any two distinct such $J$-holomorphic curves $C, C^{\prime}$ intersect only at the singular point $x$. The latter follows from the facts that (1) $C \cdot C^{\prime} \leq C_{0} \cdot C_{0}=\frac{p}{p+q}<1$, so that by the intersection formula in Theorem $3.2, C, C^{\prime}$ do not intersect at any smooth point of $X,(2)$ there is at most one such $J$-holomorphic curve containing the other singular point $x^{\prime}$ of $X$.

Next we prove that the differential of $\mathrm{Ev}$ is invertible at each point of $Z$. Clearly the differential of Ev is injective along each fiber of $Z \rightarrow \mathcal{M}$, because each $f \in \widehat{\mathcal{M}}$ is locally embedded on $\Sigma \backslash\left\{z_{\infty}\right\}$. Hence it suffices to show that for any $f \in \widehat{\mathcal{M}}$ and any $u$ in the tangent space of $\widehat{\mathcal{M}}$ at $f$ which is not tangent to the $H$-orbit through $f, u(z) \in(T X)_{f(z)}$ is not tangent to $\operatorname{Im} f$ for any $z \in \Sigma \backslash\left\{z_{\infty}\right\}$. Note that $u$, being in the tangent space of $\widehat{\mathcal{M}}$ at $f$, satisfies $D \underline{L}_{f}(u)=0$.

Now suppose to the contrary that $u$ is tangent to $\operatorname{Im} f$ at some $z \in \Sigma \backslash\left\{z_{\infty}\right\}$. We can choose complex coordinates $w_{1}, w_{2}$ on a local uniformizing system at $f(z)$ such that $\operatorname{Im} f$ is locally given by $w_{2}=0$, and $J$ equals the standard complex structure $J_{0}$ on $w_{2}=0$ (cf Lemma 1.2.2 in [15], or the corrected version of Lemma 2.5 in [14). Let $w=s+\sqrt{-1} t$ be a local holomorphic coordinate on $\Sigma$ centered at $z$, and set $\partial=\frac{\partial}{\partial w}, \bar{\partial}=\frac{\partial}{\partial \bar{w}}$. Then

$$
\underline{L}(f) \equiv d f+J \circ d f \circ j=0, \forall f \in[\Sigma ; X]
$$

can be written locally as

$$
\bar{\partial} f^{i}+a_{\bar{k}}^{i}(f) \bar{\partial} \bar{f}^{k}=0,
$$

where $f=\left(f^{1}, f^{2}\right)$, and $a_{\bar{k}}^{i}$ is a $2 \times 2$ matrix of smooth complex valued functions of $w_{1}, w_{2}$ which vanishes on $w_{2}=0$, cf [14]. Let $u_{1}, u_{2}$ be the components of $u$ in the $\frac{\partial}{\partial w_{1}}, \frac{\partial}{\partial w_{2}}$ directions, then $D \underline{L}_{f}(u)=0$ implies that

$$
\bar{\partial} u_{2}+A u_{2}+B \bar{u}_{2}=0
$$

for some smooth complex valued functions $A, B$ of $s, t$. It follows easily that $u_{2}$ satisfies

$$
\left|\Delta u_{2}\right| \leq c\left(\left|u_{2}\right|+\left|\partial_{s} u_{2}\right|+\left|\partial_{t} u_{2}\right|\right)
$$

pointwise for some constant $c>0$, where $\Delta=\partial_{s}^{2}+\partial_{t}^{2}$. Note that $u_{2}$ is not constantly zero but $u_{2}(z)=0$ by the assumption, hence by Hartman-Wintner's theorem [9],

$$
u_{2}(w)=a w^{m}+O\left(|w|^{m+1}\right)
$$

for some nonzero $a \in \mathbb{C}$ and integer $m>0$. 
Let $f_{\lambda}, \lambda \geq 0$, be a local smooth path in $\widehat{\mathcal{M}}$ starting at $f$ which is tangent to $u$ at $\lambda=0$. Then in the local coordinate system $\left\{w_{1}, w_{2}\right\}, f_{\lambda}$ is given by a pair of functions $w_{1}=f_{\lambda}^{1}(w), w_{2}=f_{\lambda}^{2}(w)$ which satisfy

$$
\left(f_{\lambda}^{1}(w), f_{\lambda}^{2}(w)\right)=\lambda\left(u_{1}(w), u_{2}(w)\right)+O\left(\lambda^{2}\right) .
$$

We introduce $F_{\lambda}(w)=\lambda^{-1}\left(f_{\lambda}^{2}(w)-\lambda a w^{m}\right)$. Then for any fixed, sufficiently small $\lambda \neq 0$, there is an $r=r(\lambda)>0$ such that $\left|F_{\lambda}(w)\right| \leq|a| r^{m}$ for all $w$ satisfying $|w| \leq r$. For any such fixed $\lambda \neq 0$, we define a sequence $\left\{w=w_{n} \mid\right.$ $\left.\left|w_{n}\right| \leq r=r(\lambda), n=1,2, \cdots\right\}$ inductively by solving

$$
F_{\lambda}\left(w_{n}\right)+a w_{n+1}^{m}=0,
$$

then $\left\{w_{n}\right\}$ has a limit $w_{0}$ in the disc $|w| \leq r=r(\lambda)$ satisfying

$$
F_{\lambda}\left(w_{0}\right)+a w_{0}^{m}=0 .
$$

But this exactly means that $f_{\lambda}^{2}\left(w_{0}\right)=0$, which in turn implies that $\operatorname{Im} f_{\lambda}$ intersects with $\operatorname{Im} f$ near $f(z)$, for any sufficiently small $\lambda \neq 0$. A contradiction.

Hence $u$ is nowhere tangent to $\operatorname{Im} f$, and the differential of $\operatorname{Ev}: Z \rightarrow X$ is injective, hence invertible by dimension counting, at each point in $Z$.

To see that Ev maps the underlying space of $Z$ onto that of $X \backslash\{x\}$, note first that the image of $\mathrm{Ev}$ is contained in $X \backslash\{x\}$ and is an open subset. The latter is because the differential of $\mathrm{Ev}$ is invertible at each point of $Z$ so that Ev induces an open map between the underlying spaces. On the other hand, the image of Ev is also closed in $X \backslash\{x\}$. To see this, suppose $\operatorname{Ev}\left(\left[\left(f_{n}, z_{n}\right)\right]\right)=f_{n}\left(z_{n}\right)$ is a sequence of points in $X \backslash\{x\}$ which converges to $p \in X \backslash\{x\}$. Since $\mathcal{M}$ is compact, a subsequence of $f_{n}$ (still denoted by $f_{n}$ for simplicity) converges in $C^{\infty}$ to a $f_{0} \in \widehat{\mathcal{M}}$ after reparametrization. If we let $z_{0}$ be a limiting point of $z_{n}$ in $\Sigma$, then $z_{0} \neq z_{\infty}$, because otherwise $p=\lim _{n \rightarrow \infty} f_{n}\left(z_{n}\right)=f_{0}\left(z_{\infty}\right)=x$, a contradiction. This implies that the image of Ev contains $p=f_{0}\left(z_{0}\right)$, therefore it is closed in $X \backslash\{x\}$. Hence $\mathrm{Ev}$ maps $Z$ onto $X \backslash\{x\}$, and thus it is a diffeomorphism from $Z$ onto $X \backslash\{x\}$.

\section{Proof of Theorem 1.1}

First of all, note that by Lemma $4.3, \mathcal{M}$ is connected, and has an orbifold point of order $p$. The latter assertion is because there exists an $f \in \widehat{\mathcal{M}}$ such that $\operatorname{Im} f$ contains the singular point $x^{\prime} \in X$, so that $f$ must be a multiply covered map. Moreover, $\mathcal{M}$ is orientable, and we shall orient $\mathcal{M}$ such that with the canonical orientation of orbifold complex line bundle on $Z$, the map Ev: $Z \rightarrow X$ is 
orientation-preserving. In order to determine the diffeomorphism type of $\mathcal{M}$ and the isomorphism class of the orbifold complex line bundle $Z \rightarrow \mathcal{M}$, we consider the family of regular neighborhoods of $x$ :

$$
N_{\epsilon} \equiv\left\{\left.\left(z_{1}, z_{2}\right)|| z_{1}\right|^{2}+\left|z_{2}\right|^{2} \leq \epsilon^{2}\right\} / \mathbb{Z}_{(p+q)}
$$

where $z_{1}, z_{2}$ are holomorphic coordinates on a local uniformizing system at $x$ in which $C_{0}$ is locally given by $z_{2}=0$ and $C_{0}^{\prime}$, the unique $J$-holomorphic curve containing both $x, x^{\prime}$, is locally given by $z_{1}=0$.

Claim There exists an $\epsilon_{0}>0$ such that for any $0<\epsilon \leq \epsilon_{0}, \partial N_{\epsilon}$ intersects transversely with each $J$-holomorphic curve in the family parametrized by $\mathcal{M}$ at a simple closed loop.

Proof For each $\lambda \in \mathcal{M}$, pick a local representative $\left(\hat{f}_{\lambda}, \rho_{\lambda}\right)$ of a member $f_{\lambda} \in \widetilde{\mathcal{M}}$ whose orbit in $\mathcal{M}$ is $\lambda$, and set $C_{\lambda} \equiv \operatorname{Im} f_{\lambda}$. Here $\rho_{\lambda}\left(\mu_{(p+q)}\right)$ acts by $\left(z_{1}, z_{2}\right) \mapsto\left(\mu_{(p+q)} z_{1}, \mu_{(p+q)}^{p} z_{2}\right)$, and $\hat{f}_{\lambda}=\left(U_{\lambda}, V_{\lambda}\right)$ for some holomorphic functions $U_{\lambda}, V_{\lambda}$ defined on $D=\{z \in \mathbb{C}|| z \mid \leq 1\}$. Observe (1) since $\mathcal{M}$ is compact, we may assume that for any sequence $\lambda_{i} \in \mathcal{M}$ converging to $\lambda_{0} \in \mathcal{M}$, there is a subsequence of $\lambda_{i}$, still denoted by $\lambda_{i}$, such that $\hat{f}_{\lambda_{i}}$ converges to $\hat{f}_{\lambda_{0}} \circ \xi$ for some holomorphic reparametrization $\xi$ of $D,(2)$ for any $C_{\lambda} \neq C_{0}, C_{0}^{\prime}$, $C_{\lambda} \cdot C_{0}=\frac{p}{p+q}$ and $C_{\lambda} \cdot C_{0}^{\prime}=\frac{1}{p+q}$, so that by the intersection formula in Theorem 3.2 , for any such a $\lambda, U_{\lambda}(z)=a_{\lambda, 1} z+\cdots, V_{\lambda}(z)=b_{\lambda, p} z^{p}+\cdots$ near $z=0$ for some $a_{\lambda, 1} \neq 0, b_{\lambda, p} \neq 0$. (For $C_{0}$ or $C_{0}^{\prime}, \hat{f}_{\lambda}(z)$ equals $\left(a_{\lambda, 1} z+\cdots, 0\right)$ or $\left(0, b_{\lambda, p} z^{p}+\cdots\right)$ near $\left.z=0.\right)$

Now for each $\lambda \in \mathcal{M}$, we write $U_{\lambda}(z)=a_{\lambda, 1} z \cdot u_{\lambda}(z), V_{\lambda}(z)=b_{\lambda, p} z^{p} \cdot v_{\lambda}(z)$ on $D$. Then there exist $0<r_{0} \leq 1,0<\delta_{0}<1$, and $c>0$, which are independent of $\lambda$, such that

$$
1-\delta_{0} \leq\left|u_{\lambda}(z)\right|,\left|v_{\lambda}(z)\right| \leq 1+\delta_{0}, \text { and }\left|d u_{\lambda}(z)\right|+\left|d v_{\lambda}(z)\right| \leq c
$$

when $|z| \leq r_{0}$. Write $z=r \exp (\sqrt{-1} \theta)$, and set

$$
\mu_{\lambda}(r, \theta) \equiv\left|U_{\lambda}(z)\right|^{2}+\left|V_{\lambda}(z)\right|^{2} .
$$

Then each $\mu_{\lambda}$ is subharmonic on $D$, and a simple calculation shows that

$$
\frac{\partial \mu_{\lambda}(r, \theta)}{\partial r}=\left|a_{\lambda, 1}\right|^{2} r\left(2\left|u_{\lambda}\right|^{2}+r \frac{\partial}{\partial r}\left|u_{\lambda}\right|^{2}\right)+\left|b_{\lambda, p}\right|^{2} r^{2 p-1}\left(2 p\left|v_{\lambda}\right|^{2}+r \frac{\partial}{\partial r}\left|v_{\lambda}\right|^{2}\right),
$$

from which it follows that there exists $0<r_{0}^{\prime} \leq r_{0}$ such that

$$
\frac{\partial \mu_{\lambda}(r, \theta)}{\partial r}>0
$$


for all $\lambda \in \mathcal{M}$ whenever $0<r \leq r_{0}^{\prime}$.

It remains to check that (1) there exists an $\epsilon_{0}>0$ such that $\mu_{\lambda}(r, \theta) \leq \epsilon_{0}^{2}$ implies $r \leq r_{0}^{\prime}$, (2) assuming the validity of (1), for any $0<\epsilon \leq \epsilon_{0}$, the intersection of $\partial N_{\epsilon}$ with each $C_{\lambda}$, which is transverse because $\frac{\partial \mu_{\lambda}}{\partial r}>0$ on $\mu_{\lambda}^{-1}(\epsilon)$ by the validity of (1), is a simple closed loop.

To see the former, note that $\mu_{\lambda}(r, \theta) \leq \epsilon_{0}^{2}$ implies

$$
r \leq \frac{2 \epsilon_{0}}{\left|a_{\lambda, 1}\right| \cdot\left|u_{\lambda}\right|+\left(\left|b_{\lambda, p}\right| \cdot\left|v_{\lambda}\right|\right)^{1 / p}}
$$

where on the other hand, it is easily seen that there exists a $c_{1}>0$ such that for any $\lambda \in \mathcal{M}$ and $|z| \leq r_{0}$,

$$
\left|a_{\lambda, 1}\right| \cdot\left|u_{\lambda}\right|+\left(\left|b_{\lambda, p}\right| \cdot\left|v_{\lambda}\right|\right)^{1 / p} \geq c_{1} .
$$

To see the latter, suppose the intersection of $\partial N_{\epsilon}$ with some $C_{\lambda}$ consists of at least two components. Then either one of them bounds a disc in $D \backslash\{0\}$, or there is an annulus in $D \backslash\{0\}$ bounded by them. In any event, $\mu_{\lambda}$ will attain its minimum on the region at an interior point of the region (note that $\mu_{\lambda}$ is subharmonic on $D)$, contradicting the fact that $\frac{\partial \mu_{\lambda}(r, \theta)}{\partial r}>0$ there. Hence the claim.

Back to the proof of Theorem 1.1. Let $E \rightarrow \mathcal{M}$ be the orbifold bundle of unit disc associated to $Z$. Then the claim above implies that $X \backslash \operatorname{int}\left(N_{\epsilon}\right)$ is diffeomorphic to $E$ for any $0<\epsilon \leq \epsilon_{0}$. In particular, $\partial E$ is diffeomorphic to $\partial N_{\epsilon}=L(p+q, p)$. Note that $\partial E \rightarrow \mathcal{M}$ defines a Seifert fibration of the lens space $L(p+q, p)$ with one singular fiber of order $p$. Moreover, the Euler number of the Seifert fibration, which equals the self-intersection of the image of the zero section of $Z$ under the map Ev: $Z \rightarrow X$, is $1+\frac{q}{p}$ because it has a positive and transverse intersection with $C_{0}$ at a smooth point of $X$. This completely determines the diffeomorphism type of $\mathcal{M}$ and the isomorphism class of $Z$.

Now observe that the same thing works for $X_{(p, q)}(R)$ as well. In particular, the isomorphism class of $Z$ is independent of $X$ and $X_{(p, q)}(R)$. Fix an $\epsilon>0$ and set $N \equiv N_{\epsilon}$. Then from the proceeding paragraph, there are decompositions $X=N \cup_{\phi_{1}} E$ and $X_{(p, q)}(R)=N \cup_{\phi_{2}} E$, where if we let $\gamma=\left\{z_{2}=0\right\} \cap \partial N$ and let $\gamma^{\prime}=C_{0} \cap \partial E$, then $\phi_{i}(\gamma)=\gamma^{\prime}, i=1,2$. Without loss of generality, we may assume $\phi_{2}=I d$ and $\gamma^{\prime}=\gamma$ by fixing an identification of $\partial E$ with $\partial N$. With this understood, we claim that $\phi_{1}$ is isotopic to the identity through a family of diffeomorphisms $\phi_{t}: \partial N \rightarrow \partial N$ such that $\phi_{t}(\gamma)=\gamma$. 
First, assuming the validity of the claim, we obtain consequently a diffeomorphism of orbifold pairs $\psi:\left(X, C_{0}\right) \rightarrow\left(X_{(p, q)}(R), C_{0}\right)$, which preserves the singular point of order $p$ in $X$ and $X_{(p, q)}(R)$. By restricting $\psi$ to the complement of a regular neighborhood of the union of the singular point of order $p$ and the suborbifold $C_{0}$, we obtain a diffeomorphism $\psi^{\prime}: W \rightarrow L(p, q) \times[0,1]$.

It remains to verify the claim that $\phi_{1}$ is isotopic to the identity through a family of diffeomorphisms $\phi_{t}: \partial N \rightarrow \partial N$ such that $\phi_{t}(\gamma)=\gamma$. To this end, let $Y$ be the complement of a regular neighborhood of $\gamma$ in $\partial N$. Then $\pi_{1}(Y)$ is generated by the image of $\pi_{1}(\partial Y)$ in $\pi_{1}(Y)$ induced by the inclusion $\partial Y \subset Y$, ie, $\pi_{1}(Y)$ is generated by the longitude and the meridian in $\partial Y \equiv T^{2}$. The diffeomorphism $\left.\phi_{1}\right|_{Y}$ induces an automorphism of $\pi_{1}(Y)$ which is unique up to conjugation. In the present case, it is clear that the automorphism of $\pi_{1}(Y)$ can be chosen to be the identity map. Hence by the theorem of Waldhausen in [19], there exists an isotopy $\phi_{t}^{\prime}: Y \rightarrow Y$ between $\left.\phi_{1}\right|_{Y}$ and $I d$. Moreover, we may assume that $\left.\phi_{t}^{\prime}\right|_{\partial Y}: T^{2} \rightarrow T^{2}$ is given by a family of linear translations, cf [5]. The latter implies particularly that $\phi_{t}^{\prime}$ can be extended to an isotopy $\phi_{t}$ from $\phi_{1}$ to $I d$ which satisfies $\phi_{t}(\gamma)=\gamma$. Hence the claim.

\section{Proof of Corollary 1.2}

By Smith's theory (cf page 43 in [1]), and by the assumption that $\rho$ is free outside of a ball, we see easily that $\rho$ is free in the complement of its fixed-point set, which consists of a single point. Then by applying (the proof of) Theorem 1.1 to the quotient space of $\rho$, it follows easily that $\rho$ is conjugate to a linear action by a diffeomorphism of $\mathbb{R}^{4}$. To see that the diffeomorphism can be made identity outside of a ball, we note that in the diffeomorphism $\psi:\left(X, C_{0}\right) \rightarrow$ $\left(X_{(p, q)}(R), C_{0}\right)$ constructed in the proof of Theorem 1.1, $\left.\psi\right|_{C_{0}}: C_{0} \rightarrow C_{0}$ is isotopic to identity, from which it follows easily.

\section{References}

[1] A Borel et al, Seminar on transformation groups, Ann. of Math. Studies 46, Prin. Univ. Press (1960)

[2] C Boyer, K Galicki, M Nakamaye, On positive Sasakian geometry, arXiv:math.DG/0104126

[3] W Chen, On a notion of maps between orbifolds: I. function spaces, II. homotopy theory, preprint 
[4] W Chen, Y Ruan, Orbifold Gromov-Witten theory, from: "Orbifolds in Mathematics and Physics", (A Adem, et al editors), Contemporary Mathematics 310, Amer. Math. Soc. Providence, RI (2002) 25-85

[5] C J Earle, J Eells, The diffeomorphism group of a compact Riemann surface, Bull. Amer. Math. Soc. 73 (1967) 557-559

[6] Y Eliashberg, On symplectic manifolds with some contact properties, J. Diff. Geom. 33 (1991) 233-238

[7] M Gromov, Pseudoholomorphic curves in symplectic manifolds, Invent. Math. 82 (1985) 307-347

[8] A Haefliger, Complexes of groups and orbihedra, from: "Group theory from a geometrical viewpoint, 26 March - 6 April 1990, ICTP, Trieste", World Scientific (1991) 504-540

[9] P Hartman, A Wintner, On the local behavior of solutions of nonparabolic partial differential equations, Amer. J. Math. 75 (1953) 449-476

[10] T Kawasaki, The index of elliptic operators over V-manifolds, Nagoya Math. J. 84 (1981) 135-157

[11] S Kobayashi, Differential Geometry of Complex Vector Bundles, Publ. of Math. Soc. Japan 15, Iwanami Shoten, Publishers and Princeton University Press (1987)

[12] E Lerman, Symplectic cuts, Math. Res. Lett. 2 (1995) 247-258

[13] P Lisca, On symplectic fillings of lens spaces, arXiv:math.SG/0312354

[14] D McDuff, The local behaviour of holomorphic curves in almost complex 4manifolds, J. Diff. Geo. 34 (1991) 143-164

[15] D McDuff, Singularities and positivity of intersections of J-holomorphic curves, with Appendix by Gang Liu, from: "Holomorphic Curves in Symplectic Geometry", (M Audin and J Lafontaine editors) Progress in Math. vol. 117, Basel-Boston-Berlin: Berkhäuser (1994) 191-215

[16] D McDuff, D Salamon, J-holomorphic Curves and Symplectic Topology, Colloquium Publications 52, AMS (2004)

[17] R S Palais, Equivalence of nearby differentiable actions of a compact group, Bull. Amer. Math. Soc. 67 (1961) 362-364

[18] T H Parker, J G Wolfson, Pseudo-holomorphic maps and bubble trees, J. Geom. Analysis 3 (1993) 63-98

[19] F Waldhausen, On irreducible 3-manifolds which are sufficiently large, Ann. of Math. 87 (1968) 56-88

[20] F W Warner, Foundations of Differentiable Manifolds and Lie Groups, Graduate Texts in Mathematics 94, Springer-Verlag (1983)

[21] R Ye, Gromov's compactness theorem for pseudo-holomorphic curves, Trans. Amer. Math. Soc. 342 (1994) 671-694 